ARTICLE

Received 3 Mar 2016 | Accepted 14 Oct 2016 | Published 7 Dec $2016 \quad$ DOl: 10.1038/ncomms13568 OPEN

\title{
Integrin $\alpha 7$ is a functional cancer stem cell surface marker in oesophageal squamous cell carcinoma
}

\author{
Xiao-Yan Ming ${ }^{1,2}$, Li Fu ${ }^{3}$ Li-Yi Zhang1,2, Yan-Ru Qin ${ }^{4}$, Ting-Ting Cao 1,3, Kwok Wah Chan ${ }^{2,5}$, Stephanie Ma ${ }^{2,6}$, \\ Dan $\mathrm{Xie}^{7} \&$ Xin-Yuan Guan ${ }^{1,2,7}$
}

Non-CG methylation has been associated with stemness regulation in embryonic stem cells. By comparing differentially expressed genes affected by non-CG methylation between tumour and corresponding non-tumour tissues in oesophageal squamous cell carcinoma (OSCC), we find that Integrin $\alpha 7$ (ITGA7) is characterized as a potential cancer stem cell (CSC) marker. Clinical data show that a high frequency of ITGA7 ${ }^{+}$cells in OSCC tissues is significantly associated with poor differentiation, lymph node metastasis and worse prognosis. Functional studies demonstrate that both sorted ITGA7 ${ }^{+}$cells and ITGA7 overexpressing cells display enhanced stemness features, including elevated expression of stemness-associated genes and epithelial-mesenchymal transition features, as well as increased abilities to self-renew, differentiate and resist chemotherapy. Mechanistic studies find that ITGA7 regulates CSC properties through the activation of the FAK-mediated signalling pathways. As knockdown of ITGA7 can effectively reduce the stemness of OSCC cells, ITGA7 could be a potential therapeutic target in OSCC treatment.

\footnotetext{
${ }^{1}$ Department of Clinical Oncology, Li Ka Shing Faculty of Medicine, The University of Hong Kong, Hong Kong 852 , China. ${ }^{2}$ Centre for Cancer Research, Li Ka Shing Faculty of Medicine, The University of Hong Kong, Hong Kong 852, China. ${ }^{3}$ Department of Pharmacology, Shenzhen Key Laboratory of Translational Medicine of Tumor and Cancer Research Centre, School of Medicine, Shenzhen University, Shenzhen 518000, China. ${ }^{4}$ Department of Clinical Oncology, The First Affiliated Hospital, Zhengzhou University, Zhengzhou 450052, China. ${ }^{5}$ Department of Pathology, Li Ka Shing Faculty of Medicine, The University of Hong Kong, Hong Kong 852, China. ${ }^{6}$ School of Biomedical Sciences, Li Ka Shing Faculty of Medicine, The University of Hong Kong, Hong Kong 852, China. 7 State Key Laboratory of Oncology in Southern China, Sun Yat-Sen University Cancer Center, Guangzhou 510060, China. Correspondence and requests for materials should be addressed to X.-Y.G. (email: xyguan@hku.hk).
} 
$\mathrm{O}$ esophageal squamous cell carcinoma (OSCC), the major histologic subtype of oesophageal cancer, is one of the most common malignancies and ranked as the sixth leading cause of cancer death worldwide ${ }^{1}$. OSCC is characterized by its remarkable geographic distribution with particularly highrisks in Northern China ${ }^{2}$. Despite advances in diagnosis and treatment of OSCC, the 5-year survival rate after curative surgery is only $20-30 \%$, mainly due to tumour metastasis, tumour recurrence and chemoresistance ${ }^{1}$. Recently, increasing evidence suggests that cancer stem cells (CSCs) represent an important subset of tumour cells that are responsible for tumour metastasis, tumour recurrence and chemoresistance ${ }^{3-6}$. CSCs are a subset of cancer cells that are biologically distinct from the others and have stem cell-like features ${ }^{7}$. CSC has now been showed to exist in different tumour types including breast cancer ${ }^{8}$, hepatocellular carcinoma ${ }^{9}$ and OSCC $^{10}$. Currently, most CSCs are marked by either cell surface markers (for example, CD90 or CD133) or functional properties (for example, site population) ${ }^{11}$. In OSCC, surface proteins CD90 (ref. 10), CD44 (ref. 12), and p75 NTR (ref. 13), have previously been used to phenotypically mark and functionally define oesophageal CSC subsets in cell lines and clinical samples. These markers can markedly enrich the oesophageal CSC subpopulation, however, the purity of CSCs is unknown. In addition, the roles of these markers in stemness regulation and CSC maintenance are rarely understood.

A recent study by Lister et al. found that nearly $25 \%$ of all methylated sites identified in embryonic stem cells (ESC) are in a non-CG context ${ }^{14}$. Non-CG methylation disappears on induced differentiation of ESCs and can be restored in induced pluripotent stem cells (iPSC) ${ }^{14}$. Another report also demonstrated that nonCG methylation appears to be confined to stem cells ${ }^{15}$. As CSCs share many common properties with stem cells ${ }^{16,17}$, we hypothesize that non-CG methylation also predominantly exists in CSCs but not in other differentiated cancer cells. To test this, we first selected 10 stemness-associated genes with differential non-CG methylation between ESC and fibroblast from Lister et al.'s report for preliminary study in OSCC cell lines and clinical specimens. Among them, integrin $\alpha 7$ (ITGA7) was chosen for further study because it was only expressed in a small group of cancer cells in both clinical specimens and cell lines. Integrins are transmembrane cell surface receptors comprised of $18 \alpha$ and $8 \beta$ subunits. Integrins directly bind components of the extracellular matrix (ECM) and provide the traction necessary for cell motility and invasion. ITGA7, the receptor for the ECM protein laminin, forms heterodimer with integrin $\beta 1$. Integrins are involved in a broad spectrum of cellular processes, including survival, proliferation, migration and invasion ${ }^{18}$. Recent studies have suggested that integrins play important roles in the regulation of stem cell-like properties and enrich CSCs from glioblastoma multiforme $(\mathrm{GBM})^{19}$, breast cancer ${ }^{20}$ and prostate cancer ${ }^{21}$.

In the present study, we find that ITGA $^{+}$cells to be sporadically expressed in clinical OSCC specimens and that the presence of ITGA7 $^{+}$cells is closely correlated with aggressive tumour behaviour and poor outcome. Functional studies demonstrate that ITGA $^{+}{ }^{+}$cells possess strong CSC properties including abilities to self-renew, differentiate and resist chemotherapy. The molecular mechanism by which ITGA7 regulates these properties is also elucidated. Our data indicate that ITGA7 is a potential CSC marker in OSCC with function in stemness regulation.

\section{Results}

ITGA7 $^{+}$cells are markedly associated with poor outcome. Expression of ITGA7 was analyzed by immunohistochemistry (IHC) on a tissue microarray (TMA) consisting of 300 paired OSCC and non-tumour clinical samples. Informative IHC results were obtained from 262 pairs of OSCCs. Non-informative samples included lost samples and unrepresentative samples, which were not included in data complication. ITGA7-expressing cells were detected in most of these informative OSCC cases, with expression ranging from 0 to $5 \%$; while ITGA7 expression could not be detected in any of the corresponding non-tumour tissues (Fig. 1a). On the basis of the frequency of ITGA7 positive cells $\left(\right.$ ITGA $^{+}$), the OSCC patients were almost equally divided into high-frequency group $(>0.6 \%, n=137,52.3 \%)$ and low-frequency group ( $\leq 0.6 \%, n=125,47.7 \%)$ (Supplementary Table 1). Association study found that the high-frequency group was significantly associated with poor differentiation (Pearson $\chi^{2}$ test, $P=0.001$ ), presence of invasion (Pearson $\chi^{2}$ test, $P=0.009$ ), advanced clinical stage (Pearson $\chi^{2}$ test, $P<0.001$ ) and lymph node metastasis (Pearson $\chi^{2}$ test, $P=0.005$; Table 1 ). Kaplan-Meier survival analysis based on this TMA data found that OSCC patients with high frequency of ITGA $^{+}$cells $(>0.6 \%)$ were significantly associated with a shorter survival time (log-rank test, $P<0.001$; Fig. 1b). Next, we used flow cytometry to detect the frequency of ITGA $^{+}$cells in 2 immortalized oesophageal epithelial cell lines (NE1 and NE3) and 10 OSCC cell lines. The result showed that the frequency of ITGA $^{+}$cells in OSCC cell lines (except EC109) was much higher than that in immortalized oesophageal epithelial cell lines (Fig. 1c; Supplementary Fig. 1a). This result was further confirmed by immunofluorescence in NE1, EC109, KYSE180 and KYSE520 cell lines (Supplementary Fig. 1b).

Non-CG methylation is mainly detected in ITGA7 ${ }^{+}$OSCC cells. Since non-CG methylation plays important roles in the transcriptional regulation of target genes in stem cells, we next investigated whether ITGA7 expression was up-regulated in this manner. Three DNA fragments within ITGA7 gene containing CG and non-CG methylation loci were selected based on epigenetic modification data between ESC and fetal fibroblasts (http://neomorph.salk.edu/human_methylome). Bisulfite genomic sequencing (BGS) analysis was performed in sorted ITGA7 ${ }^{+}$ and ITGA7 $^{-}$cells from KYSE180 and KYSE520 cell lines. The result showed that $\mathrm{ITGA}^{+}$cells displayed much higher non-CG methylation frequency than their negative counterparts, implying that ITGA7 expression might be correlated with non-CG methylation (Fig. 1d). To determine whether the expression of ITGA7 could be restored by DNA demethylation, a DNA methylation inhibitor 5-aza-2'-deoxycytidine was used to treat sorted ITGA7 $^{+}$and ITGA7 ${ }^{-}$cells. After treatment, expression of ITGA7 was significantly increased (Fig. 1e).

ITGA7 and CD90 is co-expressed in OSCC. CD90 has previously been reported by our group to phenotypically mark a functionally defined oesophageal CSC subset ${ }^{10}$. To examine whether CD90 and ITGA7 is co-expressed, dual-colour flow cytometry was performed to examine their expression in a panel of oesophageal cell lines (Supplementary Fig. 2). Our findings included: (1) the frequencies of $\mathrm{ITGA}^{+}$cells and $\mathrm{CD} 90^{+}$cells were in the same trend (Fig. 2a; Supplementary Table 2); (2) ITGA7 $^{+}$cells were much less abundant than $\mathrm{CD} 90^{+}$cells in all oesophageal cell lines; and (3) most ITGA7 ${ }^{+}$cells in OSCC cell lines were also $\mathrm{CD}^{+}{ }^{+}$(Supplementary Table 3 ). To confirm that ITGA7 was co-expressed with CD90, cryosectioned spheroids generated from KYSE520 cells were stained and analyzed by immunofluorescence. The result showed that most of ITGA7 ${ }^{+}$ cells were co-expressed with CD90 (Fig. 2b). These findings suggested that ITGA7 $^{+}$cells may represent and mark a more specific oesophageal CSC subset than CD90. To validate this, $\mathrm{CD}{ }^{+} / \mathrm{ITGA}^{+}{ }^{+}, \mathrm{CD} 0^{+} / \mathrm{ITGA}^{-}$and $\mathrm{CD} 90^{-} / \mathrm{ITGA}^{-}$cells were separated from KYSE520 cells by cell sorting, while 
CD90 ${ }^{-} / \mathrm{ITGA}^{+}$subpopulation does not exist because most ITGA7 $^{+}$cells are also CD90 ${ }^{+}$(Fig. 2c). Western blot analysis demonstrated that the expression of stemness-associated genes was markedly higher in $\mathrm{CD} 90^{+} / \mathrm{ITGA}^{+}$cells than that in $\mathrm{CD}{ }^{+} / \mathrm{ITGA}^{-}$or $\mathrm{CD}^{-} 0^{-} / \mathrm{ITGA}^{-}$cells (Fig. $2 \mathrm{~d}$ ). Spheroid formation assay also found that the self-renewal ability was significantly enhanced in $\mathrm{CD} 90^{+} / \mathrm{ITGA} 7^{+}$cells, compared

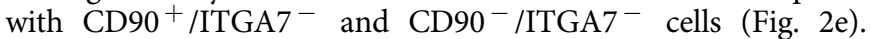
In addition, $\mathrm{CD} 90^{+} / \mathrm{ITGA}^{+}$cells were able to induce more and bigger colonies than those induced by $\mathrm{CD} 90^{+} / \mathrm{ITGA}^{-}$and $\mathrm{CD}^{-} 0^{-} / \mathrm{ITGA}^{-}$cells (Fig. $2 \mathrm{f}$ ).
ITGA7 ${ }^{+}$OSCC cells possess CSC properties. To further confirm ITGA7 ${ }^{+}$OSCC cells are CSCs, major cancer and stemness properties including expression of stemness-associated genes and abilities to self-renew, differentiate and resist chemotherapy were analyzed in ITGA7 sorted subsets isolated from KYSE180 and KYSE520 cells. ITGA7 ${ }^{+}$cells expressed higher levels of OCT3/4, SOX2, NANOG and CD90, compared with ITGA7 $^{-}$cells (Fig. 3a). Further, ITGA ${ }^{+}$cells displayed significantly enhanced ability to initiate spheroid formation and to expand in subsequent serial propagations, compared with ITGA $^{-}$cells, demonstrating their increased ability to self-renew (Fig. 3b).

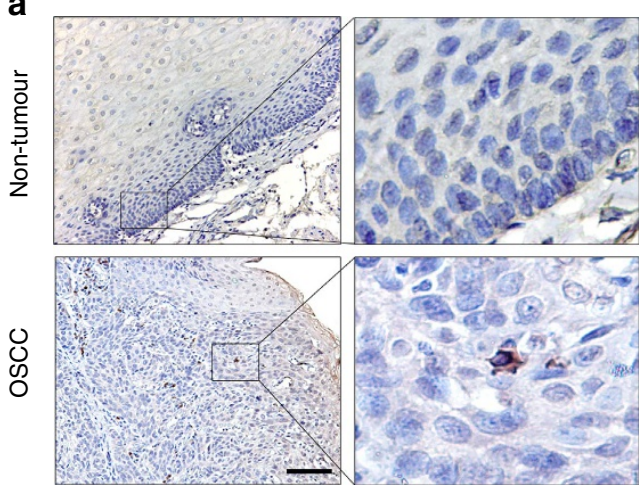

b

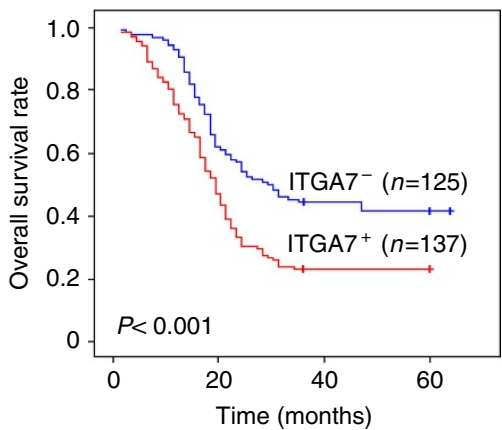

C

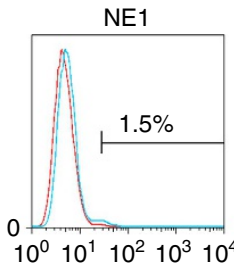
KYSE180

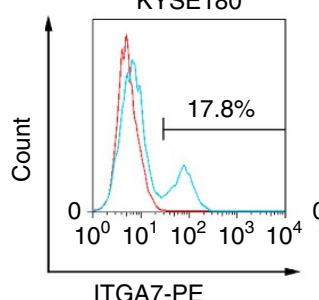

ITGA7-PE

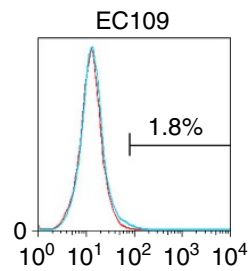

KYSE520

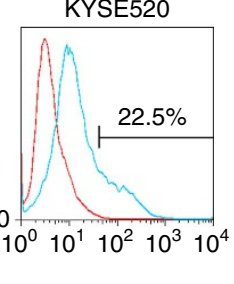

d
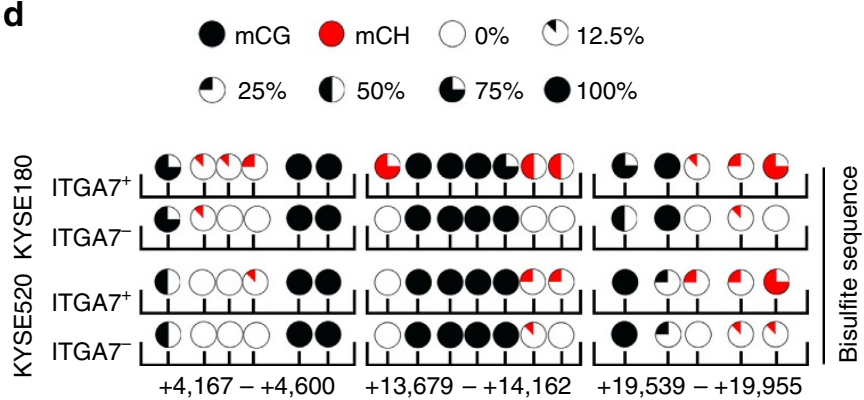

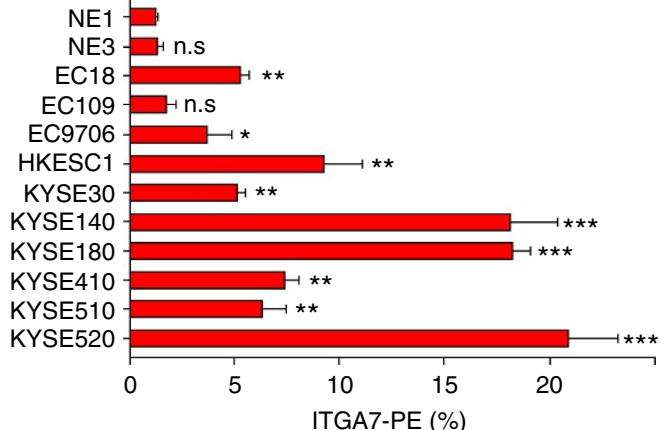

ITGA7-PE (\%)

Figure 1 | High frequency of ITGA7 ${ }^{+}$cells is significantly associated with poor outcome in OSCC. (a) Representative IHC images show that ITGA7 ${ }^{+}$ cells were scattered in OSCC tumour tissue in clinical specimen, but not in non-tumour tissue. Scale bar, $100 \mu$ m. (b) Kaplan-Meier survival analysis shows that OSCCs with high frequency of ITGA7 ${ }^{+}$cells $\left(>0.6 \%\right.$, ITGA7 $\left.{ }^{+}, n=137\right)$ had shorter survival time, compared with OSCCs with low frequency of ITGA7 ${ }^{+}$cells $\left(\leq 0.6 \%, \mathrm{ITGA7}^{-}, n=125\right)$. (c) Percentage of ITGA7 ${ }^{+}$cells detected by FACS in immortalized esophageal epithelial and OSCC cell lines. The average percentage of ITGA7 ${ }^{+}$cells, the mean \pm s.d. of three independent detections, in different cell lines was depicted in the bar chart. (d) Detection of DNA methylation in the $\mathrm{CG}$ and $\mathrm{CH}$ context $(\mathrm{H}=\mathrm{A}, \mathrm{C}$ or $\mathrm{T})$ by genomic bisulfite sequence. Non- $\mathrm{CG}$ methylation of ITGA7 preferred to occur in ITGA7 + cells isolated from KYSE180 and KYSE520. (e) qRT-PCR showed that the expression of ITGA7 was markedly increased after treated with 5-aza-2'-deoxycytidine (5-aza-dC, $50 \mu \mathrm{M})$ for 3 days. Statistics: (c,e) ANOVA with post hoc test. ${ }^{\star} P<0.05$; ${ }^{\star \star} P<0.001$; ${ }^{\star \star \star} P<0.0001 ;$ n.s., $P \geq 0.05$. 
To investigate the differentiation potential of ITGA7 $^{+}$cells, differentiation inducer all-trans retinoic acid (atRA) was used to treat ITGA7 $^{+}$and ITGA7 $^{-}$cells. Western blot analysis showed that atRA treatment could effectively induce the differentiation of ITGA7 $^{+}$cells by increasing CK13 (a marker of differentiated supra basal cells) and decreasing CK14 (a marker of oesophageal basal cell) expression. Consequently, the expression of ITGA7 and stemness-associated genes OCT3/4, SOX2 and NANOG was also decreased (Fig. 3c). However, no expression change was observed in ITGA7 $^{-}$cells after atRA treatment. This data demonstrated that ITGA7 ${ }^{+}$cells possessed differentiation ability. To check whether $\mathrm{ITGA7}^{+}$cells are more chemoresistant, chemotherapeutic reagents cisplatin and 5-fluorouracil (5-FU) were used to treat ITGA7 $^{-}$and ITGA7 $^{+}$OSCC cells. Flow cytometry analysis showed that the apoptotic/necrotic cells were significantly higher in ITGA7 $^{-}$cells compared with ITGA7 ${ }^{+}$ cells (Fig. 3d), indicating that ITGA7 $^{+}$cells were more resistant to the chemotherapeutic drugs. To further investigate the in vivo tumorigenic potential of ITGA7 $^{+}$cells, ITGA7 $^{+}$and ITGA7 ${ }^{-}$ cells were subcutaneously engrafted in NOD/SCID mice, respectively. As few as $10,000 \mathrm{ITGA}^{+}$cells were sufficient to generate visible tumours 4 months post injection, whereas, at least $100,000 \mathrm{ITGA}^{-}$cells were necessary to generate a tumour in the same mouse model (Fig. 3e; Table 2).

Knockdown of ITGA7 reduces CSC properties in OSCC cells. To further investigate if ITGA7 is a functional marker of oesophageal CSCs, we performed similar assays to examine cancer and stemness properties in OSCC cells with ITGA7 stably suppressed. Expression of ITGA7 in KYSE180 and KYSE520 cells was silenced with ITGA7-specific short hairpin RNA (shRNA). Cells treated with scrambled shRNA were used as non-target controls (NTC). After confirmation of successful knockdown with a $60 \%$ reduction by flow cytometry (Fig. 3f) and western blotting (Fig. 3g), we first detected the expression of stemnessassociated genes. Compared with cells treated with scrambled shRNA, OCT3/4, SOX2 and NANOG were down-regulated in shITGA7-treated cells (Fig. 3g). To determine whether ITGA7 knockdown could affect chemoresistant potential, cisplatin and 5-FU were used to treat shITGA7-transfected cells. The apoptotic index of shITGA7-transfected OSCC cells was significantly higher than that of control cells after a 48-h exposure to chemotherapeutic reagents (Fig. 3h; Supplementary Fig. 3). Spheroid formation assay showed that shITGA7-transfected KYSE180 and KYSE520 cells formed smaller and less spheroids, compared with control cells (Fig. 3i). In view of the importance of ITGA7 in regulating differentiation, western blot analysis was used to detect expression level of well differentiation marker CK13 and poor differentiation marker CK14 in OSCC. The result showed that ITGA7 could up-regulate CK14 and down-regulate CK13 expression (Fig. 3j). To investigate the in vivo tumorigenic potential of ITGA7 knockdown cells, scrambled shRNA and shITGA7-treated cells were subcutaneously engrafted in NOD/SCID mice, respectively. To minimize the number of mice to be used, two ITGA7-specific shRNAs-treated cells were equally mixed together as shITGA7 group. Compared with control group, knockdown of ITGA7 could effectively suppress tumour initiation (Table 3).

Ectopic overexpression of ITGA7 enhances CSC properties. As a complementary model, ITGA7 was also stably transfected into EC109 and KYSE30 cells. Expression level of ITGA7 in ITGA7-transfected cells was determined by western blotting (Fig. 4a) and flow cytometry (Fig. 4b). Western blotting results also showed that the ectopic expression of ITGA7 could enhance

\begin{tabular}{|c|c|c|c|c|}
\hline \multirow[t]{2}{*}{ Features } & \multirow[t]{2}{*}{ Total } & \multicolumn{2}{|c|}{ ITGA7 expression^ } & \multirow[t]{2}{*}{$\boldsymbol{P}_{\text {-value }}^{\dagger}$} \\
\hline & & $\begin{array}{c}\text { Low } \\
\text { frequency }\end{array}$ & $\begin{array}{c}\text { High } \\
\text { frequency }\end{array}$ & \\
\hline Sex & & & & 0.390 \\
\hline Male & 150 & $75(50 \%)$ & $75(50 \%)$ & \\
\hline Female & 112 & $50(44.6 \%)$ & $62(55.4 \%)$ & \\
\hline Age & & & & 0.593 \\
\hline$<60$ & 138 & $68(49.3 \%)$ & $70(50.7 \%)$ & \\
\hline$\geq 60$ & 124 & $57(46 \%)$ & $67(54 \%)$ & \\
\hline Differentiation & & & & $0.001^{\ddagger}$ \\
\hline Well/moderate & 195 & $105(53.8 \%)$ & $90(46.2 \%)$ & \\
\hline Poor & 67 & $20(29.9 \%)$ & $47(70.1 \%)$ & \\
\hline Tumour stage ${ }^{\S}$ & & & & $<0.001^{\ddagger}$ \\
\hline Early stage I-II & 170 & $96(56.5 \%)$ & $74(43.5 \%)$ & \\
\hline Advanced stage III-IV & 92 & $29(31.5 \%)$ & $63(68.5 \%)$ & \\
\hline Invasion & & & & $0.009 \ddagger$ \\
\hline Absent & 90 & $53(58.9 \%)$ & $37(41.1 \%)$ & \\
\hline Present & 172 & $72(41.9 \%)$ & $100(58.1 \%)$ & \\
\hline Lymph-node metastasis & & & & $0.005^{\ddagger}$ \\
\hline Absent & 142 & 79 (55.6\%) & $63(44.4 \%)$ & \\
\hline Present & 120 & $46(38.3 \%)$ & $74(61.7 \%)$ & \\
\hline
\end{tabular}

expression of stemness-associated genes (Fig. 4a). To examine whether the ectopic expression of ITGA7 could affect chemoresistant potential, cisplatin and 5-FU were used to treat ITGA7-transfected cells. The apoptotic index of ITGA7transfected cells was significantly lower than that of control cells, which is similar to sorted ITGA7 ${ }^{+}$cells (Fig. 4c). Spheroid formation assay showed that ITGA7-transfected cells formed significantly larger and more spheroids, compared with control cells (Fig. 4d). Ectopic overexpression of ITGA7 resulted in a marked decrease in the well-differentiated marker CK13. Note EC109 did not express CK13 and neither cell lines expressed CK14, and thus deregulation in expression could not be tested (Fig. $4 \mathrm{e}$ ).

The tumorigenic ability of ITGA7 was assessed by foci formation and soft agar assays. Compared with control cells, ITGA7-expressing cells displayed higher foci formation frequencies (Fig. 4f) and enhanced colony forming abilities in soft agar (Fig. 4g). To further investigate the in vivo tumorigenic ability of ITGA7, ITGA7-transfected cells and control cells were subcutaneously injected into the right and left dorsal flank of NOD/SCID mice, respectively. The result found that ITGA7-overexpressed EC109 and KYSE30 cells displayed significantly stronger tumour initiation ability than their control cells (Fig. 4h; Table 3).

ITGA7 promotes metastasis by inducing EMT. As high frequency of ITGA7 $^{+}$cells was clinically associated with tumour invasion and metastasis, the effect of ITGA7 on metastasis was further studied. Cell migration and invasion assays showed that ITGA7 $^{+}$cells could significantly promote cell migration and invasion abilities, as compared with ITGA $^{-}$cells (Fig. 5a; Supplementary Fig. 4a). To investigate the effect of ITGA7 on cell mobility, wound healing assay was performed in ITGA7 upregulated cells and down-regulated cells. The result showed that ITGA7 could markedly enhance tumour cell mobility (Supplementary Fig. 4b). Cell migration and invasion assays also displayed that ITGA7-transfected cells could promote cell migration and invasion abilities, as compared with control cells 


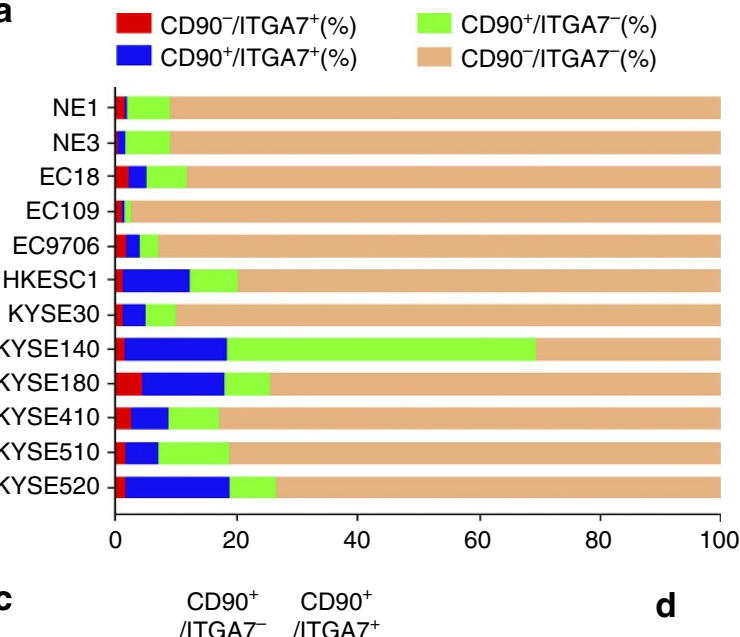

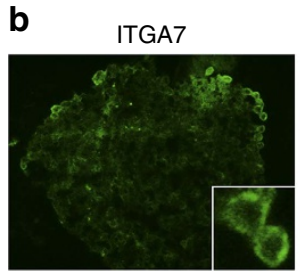

DAPI

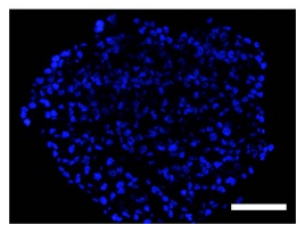

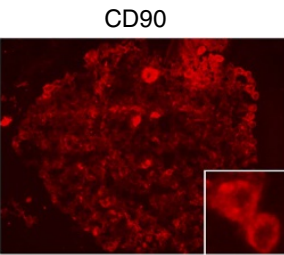

Merge

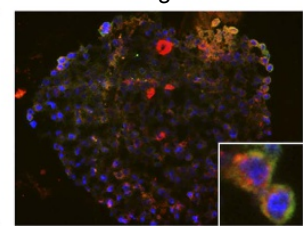

C

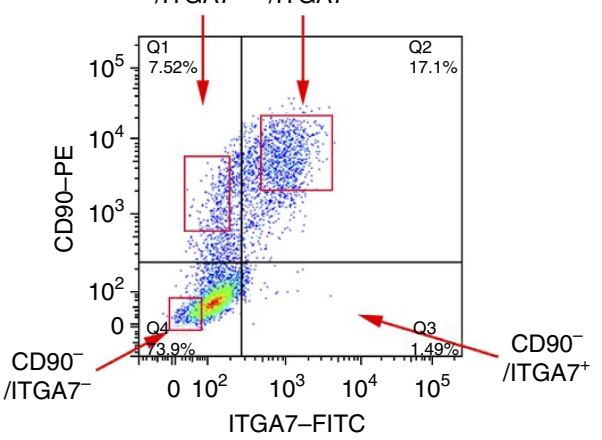

e

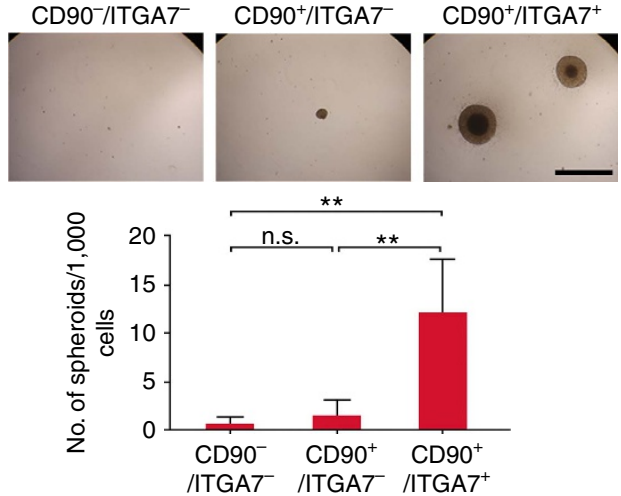

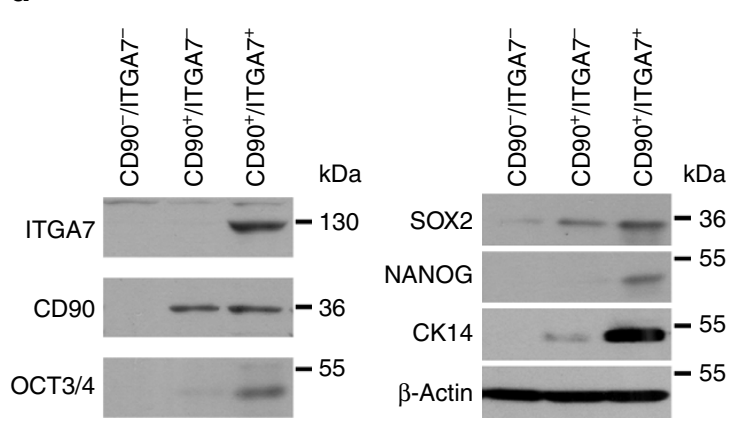

f
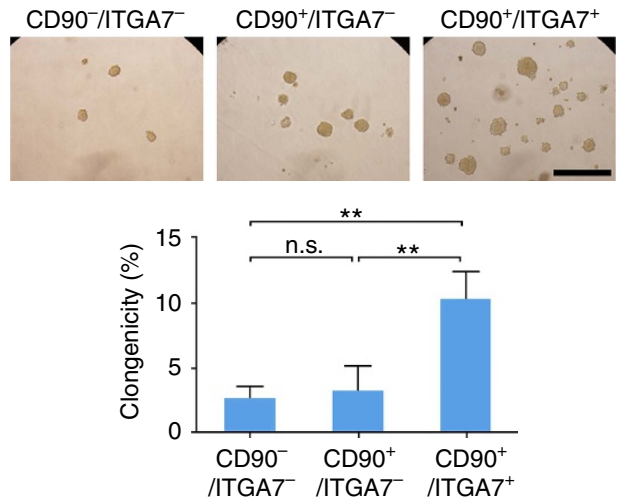

Figure 2 | ITGA7 and CD90 is co-expressed in OSCC and marks a more tumorigenic OSCC subpopulation. (a) Summary of the overlap of ITGA7 ${ }^{+}$and $\mathrm{CD}^{+} 0^{+}$cells in esophageal cell lines detected by dual-colour flow cytometry. (b) Representative dual-colour immunofluorescence analyses of cryosectioned spheroids generated from KYSE520 showing the co-localization of ITGA7 (green) and CD90 (red). Scale bar, $50 \mu \mathrm{m}$. (c) Representative dual-colour flow cytometry analysis of KYSE520 cells for ITGA7 and CD90. The cells (dot plots) were divided into four quadrants as indicated and collected for each quadrant as gated in the red boxes. $\mathrm{CD90}-/ \mathrm{ITGA7}{ }^{+}$subpopulation does not exist because most ITGA7 ${ }^{+}$cells are also $\mathrm{CD} 90^{+}$. Freshly isolated subpopulations were detected by western blotting analysis (d), and subjected to spheroid formation assay (e, Scale bar, $500 \mu \mathrm{m}$ ) and soft agar colony formation assay $(\mathbf{f}$, Scale bar, $500 \mu \mathrm{m})$. Data are represented as mean \pm s.d. of three separate experiments. Statistics: (e,f) ANOVA with post hoc test.

${ }^{\star \star} P<0.001 ;$ n.s., $P \geq 0.05$.

(Supplementary Fig. 4c). Once endogenous ITGA7 was silenced by shRNA, the abilities of cell migration and invasion were significantly inhibited (Fig. 5b). To further investigate the effect of ITGA7 on tumour metastasis, a lymph node metastasis animal model was performed by injecting ITGA7-transfected KYSE30 cells into right hind foot-pad of NOD/SCID mice. Empty vectortransfected cells were used as controls. In the lymph node metastasis model, popliteal lymph nodes, which represent the sentinel lymph node for the model, were examined ${ }^{22}$. Swollen popliteal lymph nodes were observed in all seven mice injected with ITGA7-transfected cells, and lymph node metastasis was confirmed by $\mathrm{H} \& \mathrm{E}$ staining in all seven mice. Only one-seventh of mice injected with control cells displayed tumour metastasis (Fig. 5c).

Morphological change from round shape to spindle-like shape was observed in ITGA7-transfected cells, suggesting that these cells may undergo epithelial-mesenchymal transition (EMT) (Fig. 5d). To investigate whether this morphological change was caused by EMT, immunofluorescence was performed to test some representative epithelial (E-cadherin and $\beta$-catenin) and mesenchymal (fibronectin and vimentin) markers. Compared with control cells, expression of E-cadherin was decreased in 

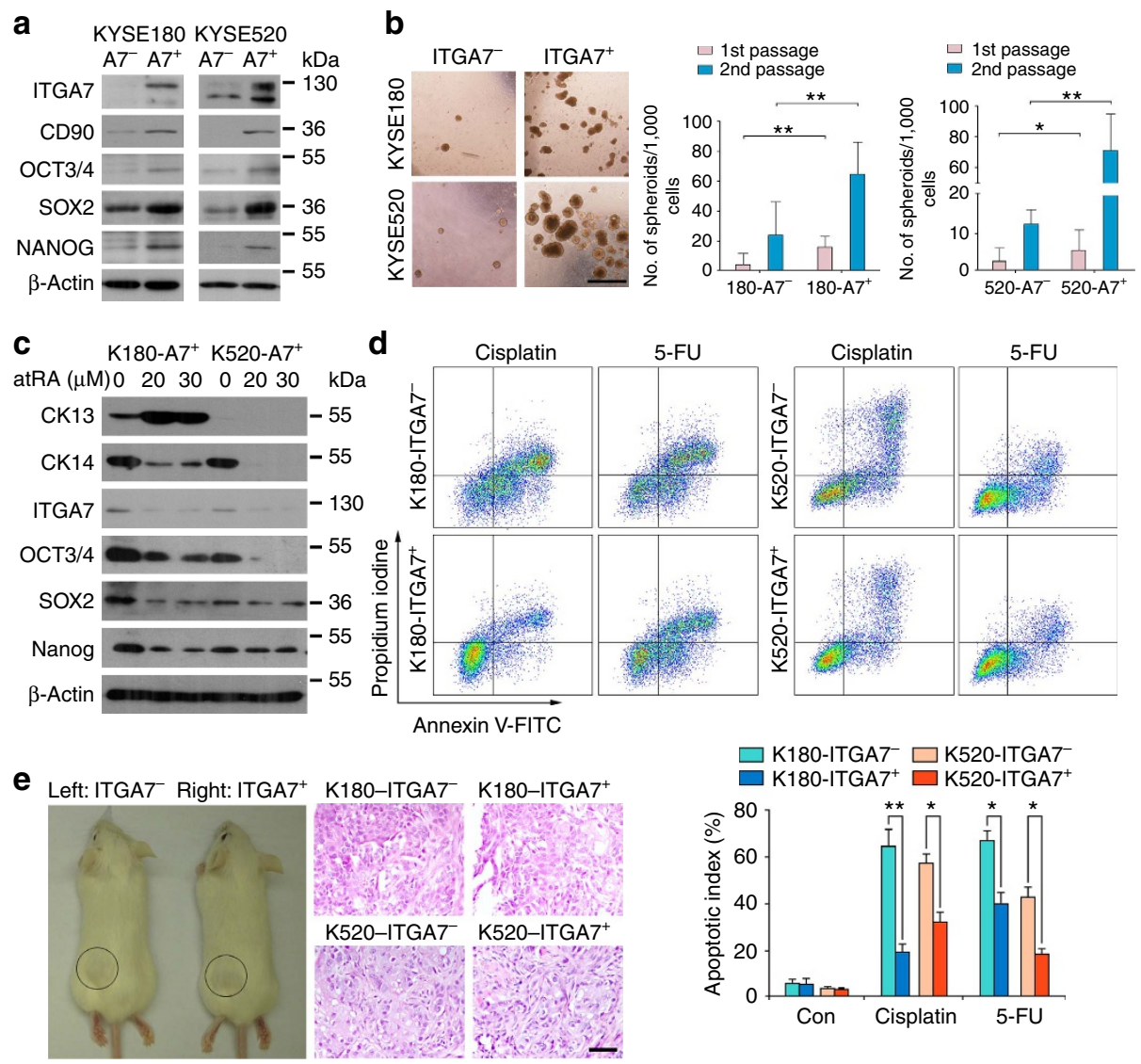

f
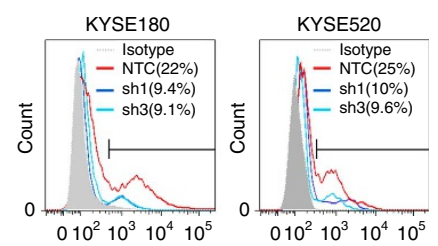

$\mathbf{g}$

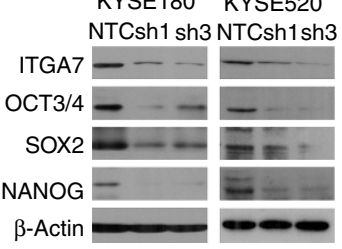

i
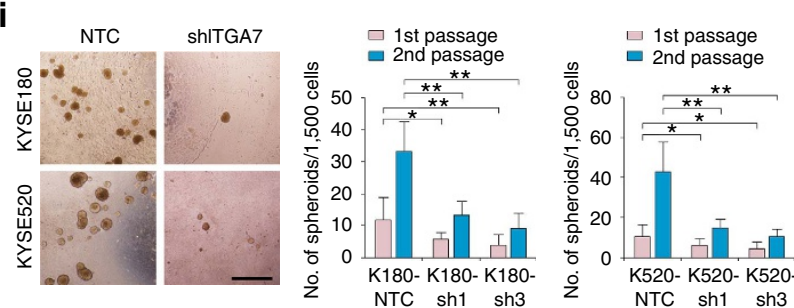

h

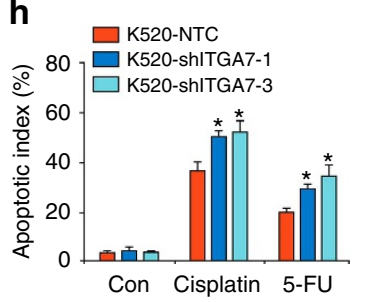

j

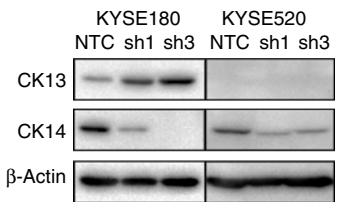

Figure 3 | ITGA7 ${ }^{+}$OSCC cells possess the CSC properties, while knockdown of ITGA7 reduces CSC properties. (a) FACS sorted ITGA7 ${ }^{+}$cells from KYSE180 and KYSE520 showed higher expression level of stemness-associated genes and CD90, compared with ITGA7 ${ }^{-}$cells. $\beta$-Actin was used as a loading control. (b) By spheroid formation assay, the self-renewal ability was enhanced in ITGA7 ${ }^{+}$cells. Secondary spheroids formed from dissociating spheroids also demonstrated enhanced serial spheroid-forming capacity. The values indicate the mean \pm s.d. of two separate experiments with 24 wells per condition. Scale bar, $500 \mu \mathrm{m}$. (c) On induced by differentiation drug atRA for 5 days, expression of CK13 and CK14 was increased (no CK13 expression in KYSE520) and decreased in ITGA7 ${ }^{+}$cells, respectively. Decreased expression of ITGA7 and stemness-associated genes was also detected in ITGA7 ${ }^{+}$ cells. (d) Representative of apoptosis analyses showing that ITGA7 ${ }^{+}$cells were more resistant to chemotherapeutic drugs, compared with ITGA7 ${ }^{-}$cells. The apoptotic index was summarized in the bar chart and the value indicated the mean \pm s.d. of three independent experiments. (e) Representative of tumours formed in NOD/SCID mice injected with the indicated cells. H\&E staining of tumours confirmed a malignant phenotype. Scale bar, $50 \mu \mathrm{m}$. $(\mathbf{f}, \mathbf{g})$ shRNA-mediated ITGA7 silencing was confirmed by FACS and western blot analysis. The expression of stemness-associated genes was decreased in shITGA7-transfected cells, compared with non-target control (NTC). (h) The summary of apoptotic index showing that ITGA7 silencing decreased the chemoresistance of KYSE520 cells. The value indicated the mean \pm s.d. of three independent experiments. (i) Spheroid formation assay showed that the self-renewal ability was decreased in ITGA7 silencing cells. The values indicate the mean \pm s.d. of two separate experiments with 24 wells per condition. Scale bar, $500 \mu \mathrm{m}$. (j) Knockdown of ITGA7 promoted the differentiation of OSCC cells. CK13 and CK14 is a well and poor differentiation marker in OSCC, respectively. Statistics: (b, d) Student $t$-test. $(\mathbf{h}, \mathbf{i})$ ANOVA with post hoc test. ${ }^{\star} P<0.05$; ${ }^{\star \star} P<0.001$. 
ITGA7-KYSE30 cells. Although the expression level of $\beta$-catenin was similar, its nuclear accumulation was observed in ITGA7transfected cells. Expression of mesenchymal markers fibronectin and vimentin was elevated in ITGA7-KYSE30 cells (Fig. 5d). The immunofluorescence result was further confirmed by western blot analysis. Compared with negative counterparts, the expression level of mesenchymal markers was markedly increased in ITGA7transfected cells and $\mathrm{ITGA7}^{+}$cells, while the expression of E-cadherin was decreased in ITGA7-transfected KYSE30 cells and KYSE520 ITGA7 ${ }^{+}$cells, no significant difference was observed in ITGA7-transfected EC109 cells and KYSE180 ITGA7 $^{+}$cells; maybe the regulation of E-cadherin is cell line specific (Fig. 5e). Conversely, ITGA7 knockdown displayed a reverse tread in expression of epithelial and mesenchymal markers (Fig. 5f).

ITGA7 drives CSC features via the FAK/MAPK/ERK signalling. As focal adhesion kinase (FAK) is a major downstream target activated by integrins, we next studied whether ITGA7 can activate FAK and the corresponding FAK/MAPK/ERK pathway. Compared with ITGA7 $^{-}$cells, ITGA7 ${ }^{+}$cells displayed an enhanced phosphorylation of FAK, Src and c-Raf with a concomitant increase in the downstream phosphorylation of MEK1/2 and ERK (Fig. 6a). To confirm the FAK/MAPK/ERK pathway could be activated by ITGA7, we also tested the expression of activated kinases in this signalling cascade in ITGA7-transfected EC109 and KYSE30 cells. The results demonstrated that the FAK/MAPK/ERK pathway could be activated by ITGA7 (Fig. 6b). Consistently, decreased phosphorylation of FAK, Src, c-Raf, MEK1/2 and ERK was observed when ITGA7 was silenced (Fig. 6c).

To further confirm the effect of ITGA7 on cancer stemness is through the FAK-mediated pathway, we studied whether a specific FAK autophosphorylation inhibitor Y15 (1,2,4,5-benzenetetraamine tetrahydrochloride) could abolish ITGA7-induced tumorigenicity and metastasis. Addition of Y15

\begin{tabular}{|c|c|c|c|c|}
\hline Cells injected & $\begin{array}{l}\text { KYSE180 } \\
\text { ITGA7 }^{+}\end{array}$ & $\begin{array}{l}\text { KYSE180 } \\
\text { ITGA7 }^{-}\end{array}$ & $\begin{array}{c}\text { KYSE520 } \\
\text { ITGA7 }^{+}\end{array}$ & $\begin{array}{c}\text { KYSE520 }^{\text {KY }} \\
\text { ITGA7 }^{-}\end{array}$ \\
\hline 5,000 & $1 / 4$ & $0 / 4$ & $0 / 4$ & $0 / 4$ \\
\hline 10,000 & $1 / 4$ & $0 / 4$ & $1 / 4$ & $0 / 4$ \\
\hline 50,000 & $3 / 4$ & $0 / 4$ & $2 / 4$ & $0 / 4$ \\
\hline 100,000 & $4 / 4$ & $1 / 4$ & $4 / 4$ & $0 / 4$ \\
\hline 300,000 & $4 / 4$ & $3 / 4$ & $4 / 4$ & $2 / 4$ \\
\hline $\begin{array}{l}\text { Tumour-initiating } \\
\text { frequency }{ }^{\star}\end{array}$ & $1 / 28,733$ & $1 / 242,532$ & $1 / 46,830$ & $1 / 770,323$ \\
\hline$P$ value ${ }^{\star}$ & \multicolumn{2}{|c|}{$P=0.0003$} & \multicolumn{2}{|c|}{$P<0.0001$} \\
\hline
\end{tabular}

effectively reduced FAK activity and ERK phosphorylation in ITGA7-transfected KYSE30 cells (Fig. 6d). Functional assays also found that the treatment of Y15 could significantly inhibit spheroid formation (Fig. 6e), cell migration (Fig. 6f) and invasion abilities (Fig. 6g), and down-regulated expression of OCT3/4, SOX2 and NANOG (Fig. 6h), as compared with untreated cells. Moreover, to investigate whether MAPK/ERK is the downstream player of ITGA7, we also treated ITGA7-KYSE30 cells with MEK1/2 inhibitor U0126. After treatment, the phosphorylation of MEK1/2 and ERK decreased markedly in ITGA7-treasfected cells (Supplementary Fig. 5a). Functional study showed that U0126 treatment significantly suppressed foci formation (Supplementary Fig. 5b), cell migration (Supplementary Fig. 5c) and invasion abilities (Supplementary Fig. 5d), as compared with untreated cells. Taken together, these results suggested that ITGA7 functionally conferred cancer stemness features in OSCC via activation of the FAK/MAPK/ERK pathway.

Chemotherapy enriches ITGA7 ${ }^{+}$CSCs in clinical OSCC tumours. To obtain further evidence in support of our hypothesis that ITGA7 ${ }^{+}$cells confer chemoresistance in OSCC tumours, we established a chemoresistant OSCC xenograft model by treating KYSE520 subcutaneously developed tumours in NOD/SCID mice with varying doses of cisplatin from 0 to $5 \mathrm{mg} \mathrm{kg}^{-1}$. Such treatment resulted in variable tumour inhibition among the xenografts, in a dose-dependent trend. From the dose-response curve, we found that the dosage of 2.5 or $5 \mathrm{mg} \mathrm{kg}^{-1}$ cisplatin could effectively shrink the tumour size (Fig.7a). Interestingly, IHC result showed that the frequency of $\mathrm{ITGA}^{+}$cells was markedly enriched in cisplatin-treated tumours (Fig. 7b). Further, cisplatin-treated OSCC xenografts also displayed enhanced expression of stemness-associated genes as well as ITGA7 in a dose-dependent manner (Fig. 7c). Consistently, OSCC cells derived from chemoresistant group exhibited markedly enhanced ability to form spheroids, compared with the tumour cells from control group (Fig. 7d).

We next studied whether chemotherapy could enrich ITGA $7^{+}$ cells in clinical OSCC specimens by IHC staining. To fulfil this purpose, 22 and 28 clinical OSCC samples were collected from patients that had or had not undergone chemotherapy before esophagectomy, respectively. Percentage of ITGA7 $^{+}$cells $(5.64 \pm 4.15 \%)$ was significantly enriched in tumours treated with chemotherapy, as compared with tumours without $(0.64 \pm 0.74 \%)$ (Fig. 7e). This finding suggests that ITGA ${ }^{+}$cells possess strong chemoresistant ability that causes their enrichment in OSCC tumours after chemotherapy.

ITGA7 inhibits apoptosis via activating FAK/Akt signalling. To investigate the underlying mechanism of ITGA7-mediated chemoresistance, we studied FAK downstream signalling pathway PI3K/Akt, a well-known pathway that mediates cell survival.

\begin{tabular}{|c|c|c|c|c|}
\hline Cells injected & $\begin{array}{l}\text { KYSE180 } \\
\text { NTC versus shA7 }\end{array}$ & $\begin{array}{l}\text { KYSE520 } \\
\text { NTC versus ShA7 }\end{array}$ & $\begin{array}{l}\text { EC109 } \\
\text { EV versus ITGA7 }\end{array}$ & $\begin{array}{l}\text { KYSE30 } \\
\text { EV versus ITGA7 } \\
\end{array}$ \\
\hline 50,000 & $1 / 4$ versus $1 / 4$ & $1 / 4$ versus $0 / 4$ & $0 / 4$ versus $3 / 4$ & $0 / 4$ versus $3 / 4$ \\
\hline 100,000 & $4 / 4$ versus $2 / 4$ & $3 / 4$ versus $1 / 4$ & $1 / 4$ versus $3 / 4$ & $1 / 4$ versus $3 / 4$ \\
\hline 300,000 & $4 / 4$ versus $3 / 4$ & $4 / 4$ versus $2 / 4$ & $3 / 4$ versus $4 / 4$ & $4 / 4$ versus $4 / 4$ \\
\hline
\end{tabular}

*Limiting dilution analysis. 
a

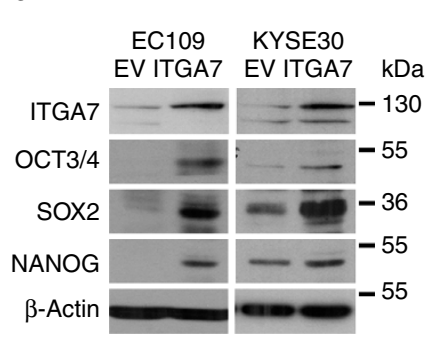

b

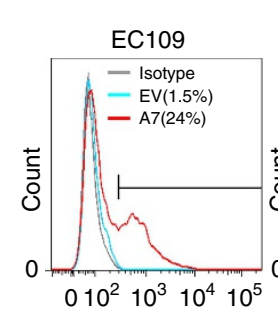

c

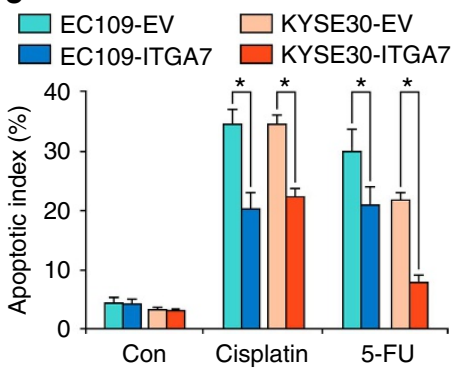

d

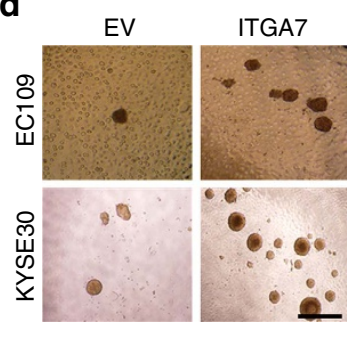

f
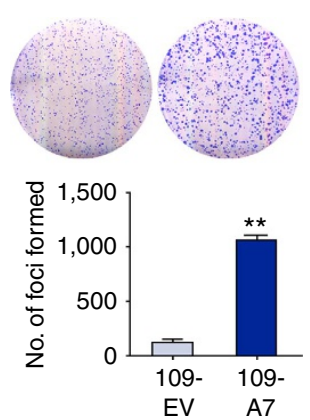
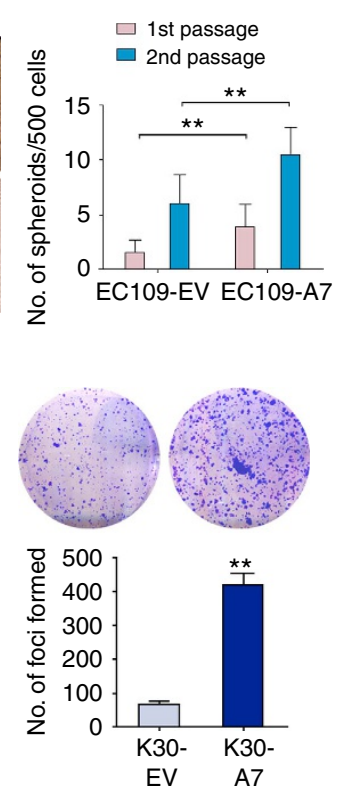

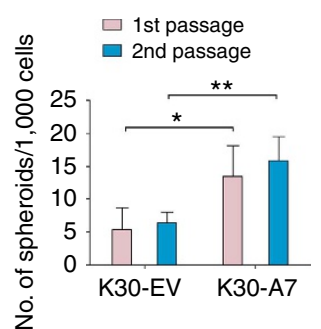

g
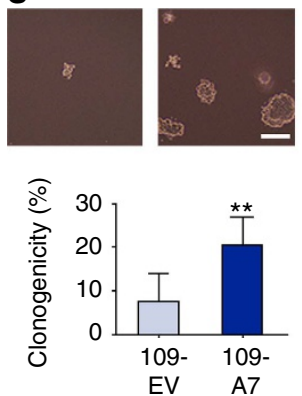

e

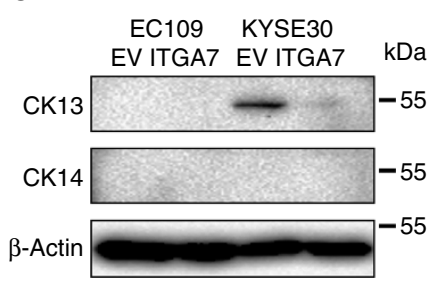

h
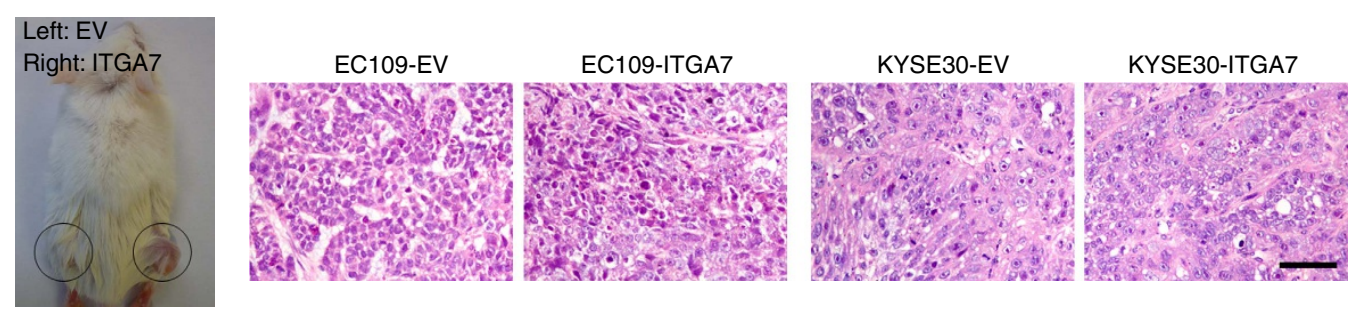

Figure 4 | Ectopic overexpression of ITGA7 enhances CSC properties in OSCC cells. (a) Expression of ITGA7 and stemness-associated genes was detected by western blotting in ITGA7- and empty vector (EV)-transfected cells. $\beta$-Actin was used as a loading control. (b) The percentage of ITGA7 ${ }^{+}$cells was compared by FACS between ITGA7- and EV-transfected cells. (c) By apoptosis assay, the anti-apoptotic ability of EC109 and KYSE30 was enhanced after ITGA7 transfection. The apoptotic index was calculated from three independent experiments and presented as mean $\pm \mathrm{s}$.d. (d) Spheroid formation assay shows the self-renewal ability was enhanced in ITGA7-transfected cells. The values indicate the mean \pm s.d. of two separate experiments with 24 wells per condition. Scale bar, $500 \mu \mathrm{m}$. (e) Expression of CK13 and CK14 was detected by western blot analysis in ITGA7-transfected and control cells. $(\mathbf{f}, \mathbf{g})$ Representative images and summaries of foci formation ( $\mathbf{f})$ and soft agar assays ( $\mathbf{g}$, Scale bar,200 $\mu$ m) performed in ITGA7-transfected and control cells. The values indicate the mean \pm s.d. of three independent experiments. (h) Representative of tumours formed in NOD/SCID mice injected with the indicated cells. H\&E staining of tumours confirmed a malignant phenotype. Scale bar, $50 \mu \mathrm{m}$. Statistics: $(\mathbf{c}, \mathbf{d}, \mathbf{f}, \mathbf{g})$ Student $t$-test. ${ }^{\star} P<0.05 ;{ }^{\star \star} P<0.001$.

Activated Akt can phosphorylate a wide variety of substrate proteins to maintain the integrity of the mitochondrial membrane, which blocks cytochrome $c$ release and subsequent caspase activation and apoptosis. Therefore, we studied whether ITGA7 could inhibit apoptosis via the FAK/PI3K/Akt signalling. The result found that phosphorylation of FAK and Akt was increased in ITGA7 ${ }^{+}$OSCC cells and ITGA7-transfected cells on cisplatin stimulation, as compared with control cells (Fig. 7f). As a result, ITGA7 $^{+}$cells and ITGA7-transfected cells maintained a high inner mitochondrial transmembrane potential $\left(\Delta \Psi_{\mathrm{m}}\right)$ (shown in red fluorescent), whereas most of the control cells underwent a proapoptotic mitochondrial permeability transition (Fig. 7g). ITGA7-induced Akt phosphorylation protected the mitochondrial membrane from cisplatin-induced collapse, thereby blocking the release of cytochrome $c$ into the cytoplasm, and the cleavages of caspase-9, caspase-3 and PARP (Fig. 7f). The anti-apoptotic phenotype and Akt phosphorylation could be effectively reversed when ITGA7 was silenced in KYSE520 cells. Reduced phosphorylated Akt in ITGA7 silenced cells led to $\Delta \Psi_{\mathrm{m}}$ collapse (Fig. 7g). As a result, cleaved forms of caspase-9, caspase- 3 and PARP increased in ITGA7 silenced cells compared with control cells (Fig. 7f). 
a

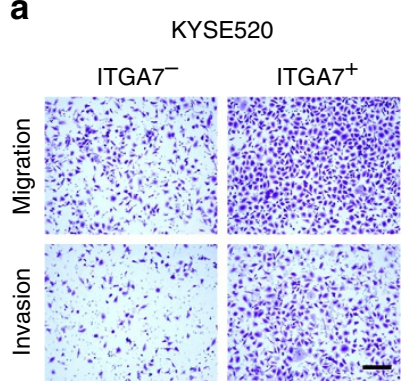

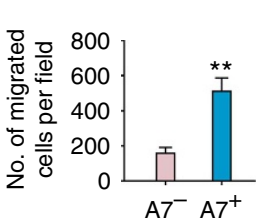

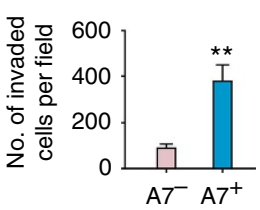

b

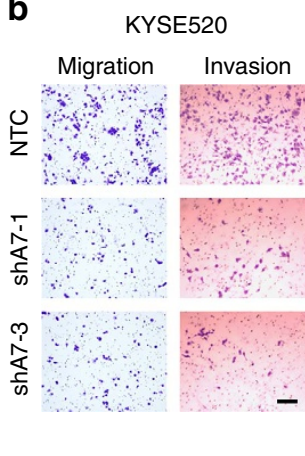

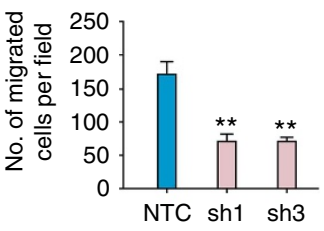

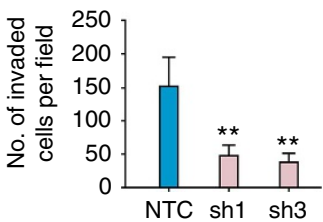

c
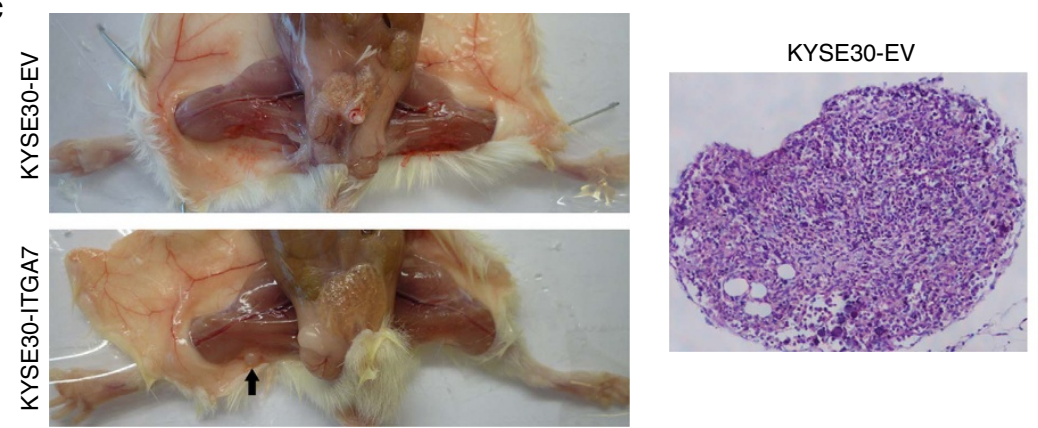

KYSE30-ITGA7

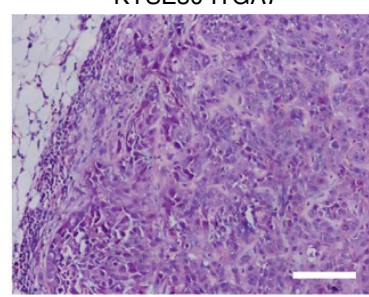

d
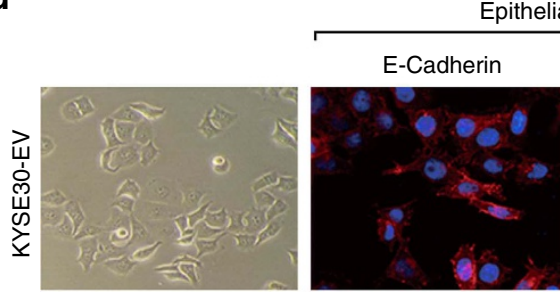

Epithelial markers
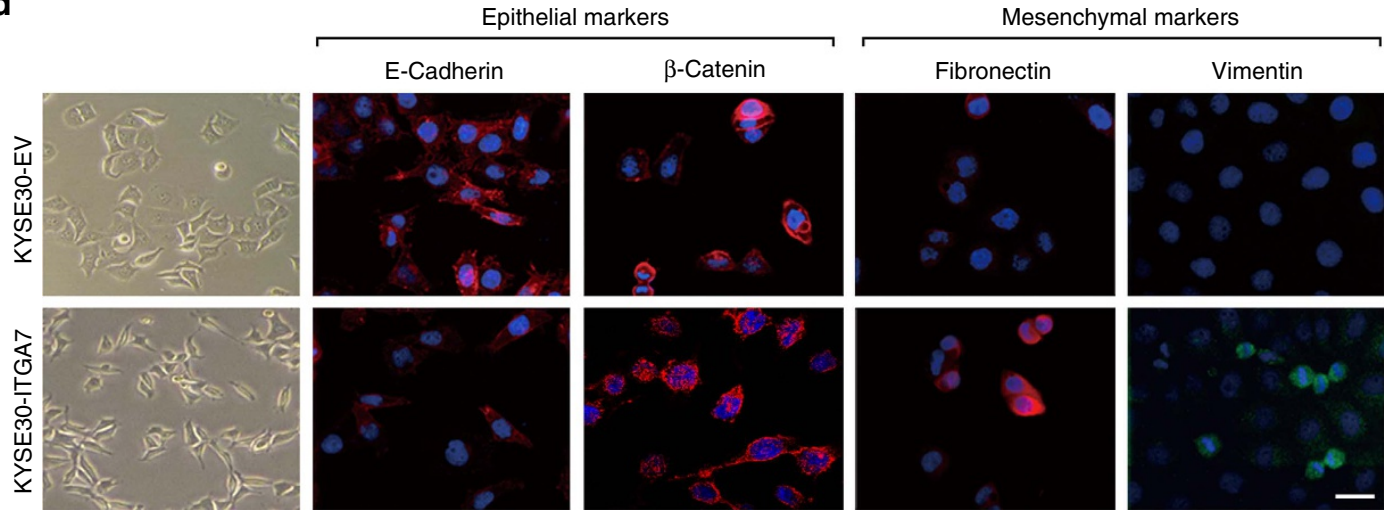

e
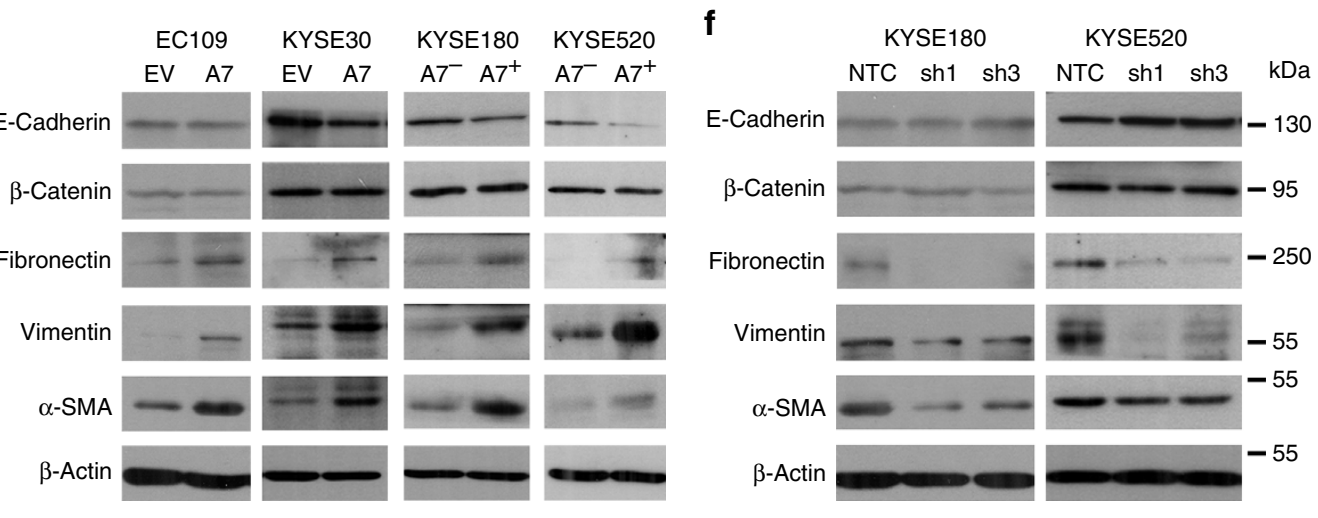

KYSE520

Figure 5 | ITGA7 promotes metastasis by inducing EMT. (a) Representatives and summary of migration and invasion assays showing that ITGA7 ${ }^{+}$cells exhibited enhanced migration and invasion abilities. The values expressed as the mean \pm s.d. of three independent experiments. Scale bar, $200 \mu \mathrm{m}$.

(b) Representatives and summary of migration and invasion analyses showing that knockdown of ITGA7-suppressed cell motility of KYSE520, compared with their control cells. The values expressed as the mean \pm s.d. of three independent experiments. Scale bar, $200 \mu \mathrm{m}$. (c) Representatives of lymph node metastasis formed in NOD/SCID mice injected with ITGA7- or EV-transfected KYSE30 cells. Black arrow indicates the swollen popliteal lymph node. Lymph nodes invaded by tumour cells were confirmed by H\&E staining (Scale bar, $100 \mu \mathrm{m}$ ). (d) Cell morphology of K30-ITGA7 and K30-EV cells. Epithelial markers (E-cadherin and $\beta$-catenin) and mesenchymal markers (fibronectin and vimentin) were detected by immunofluorescence staining (red: E-cadherin, $\beta$-catenin and fibronectin; green: vimentin; blue: nuclear DAPI staining; scale bar, $50 \mu \mathrm{m})$. (e,f) Expression of EMT markers in indicated cells was detected by western blot analysis. $\beta$-Actin was used as a loading control. Statistics: (a) Student $t$-test. (b) ANOVA with post hoc test. ${ }^{\star \star} P<0.001$. 


\section{Discussion}

So far, the CSCs have been functional defined in a wide variety of solid tumours using extensive markers, including brain, breast, colon, liver and prostate cancers. However, there are several important questions that remain understudied. One question is how sensitive and specific these CSC markers are in marking a

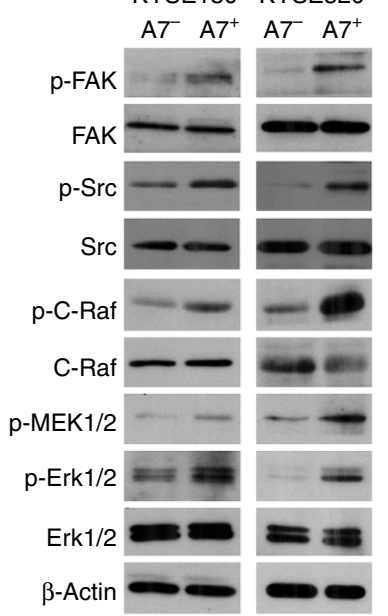

b

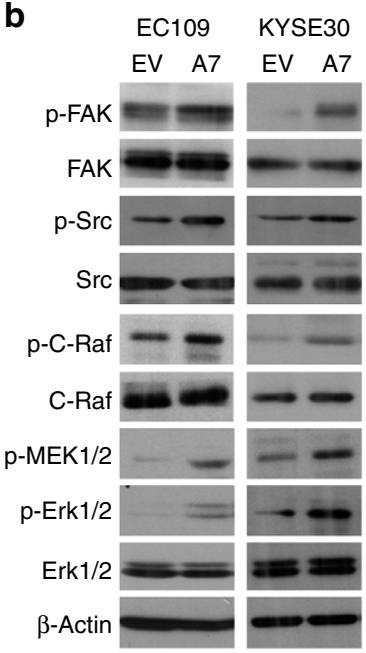

C

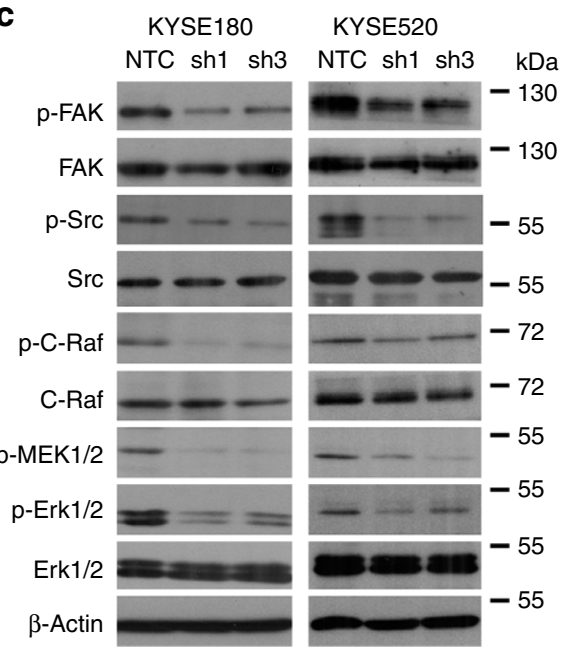

d

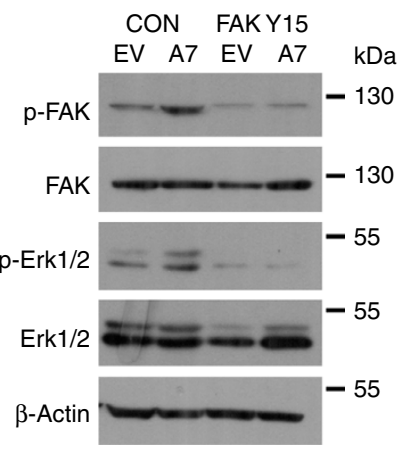

e

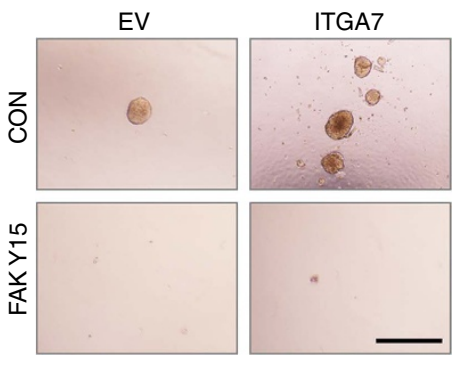

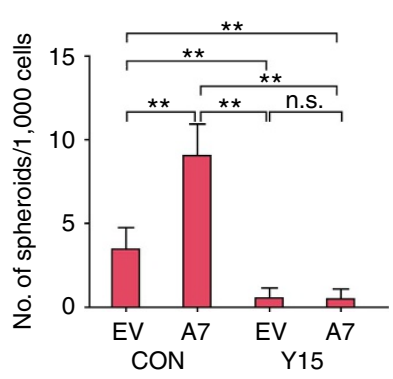

f

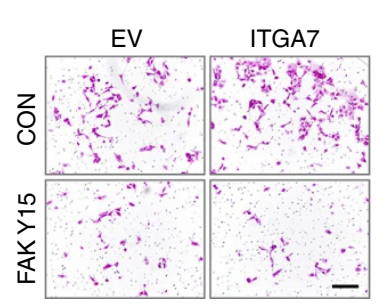

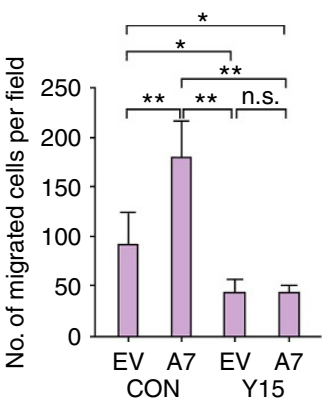

g
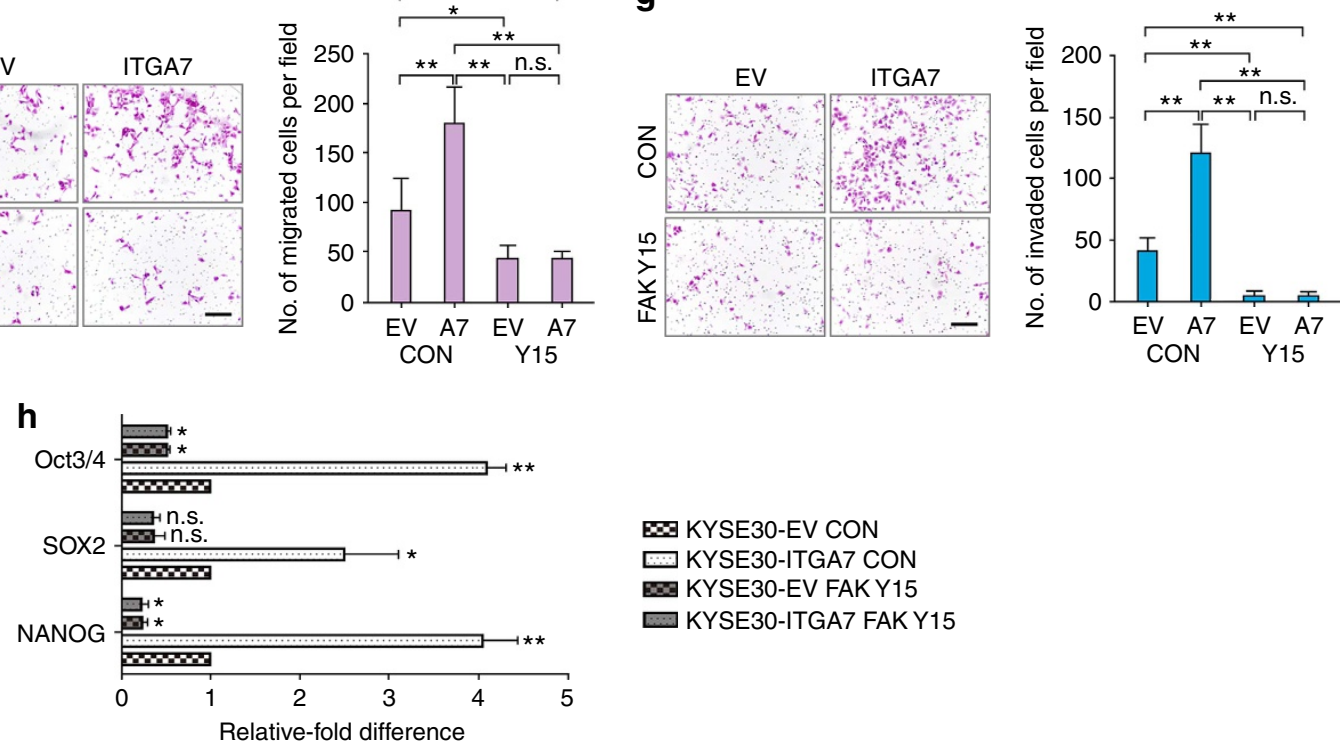

Figure 6 | ITGA7 drives cancer stemness features in OSCC via the FAK/MAPK/ERK signalling. (a-c) Western blot analyses showed that the FAK/ MAPK/ERK pathway was activated in sorted ITGA7 ${ }^{+}$cells and ITGA7-transfected cells, while inhibited in ITGA7 silencing cells. $\beta$-Actin was used as a loading control. (d) Western blot analyses showed that the phosphorylation of FAK and downstream player Erk1/2 was significantly blocked in ITGA7transfected KYSE30 cells after treatment with $7.5 \mu \mathrm{M}$ FAK inhibitor Y15. Functional assays demonstrated that Y15 treatment could effectively inhibit spheroid formation ability (e; $500 \mu \mathrm{m}$ scale bar), cell migration ability (f; $200 \mu \mathrm{m}$ scale bar), cell invasion ability (g; $200 \mu \mathrm{m}$ scale bar) and the expression of stemness-related genes in ITGA7-transfected cells $(\mathbf{h})$. The number of spheroids and migrated or invaded cells was calculated from three independent experiments and depicted as the mean \pm s.d. in the bar chart. Statistics: (e-h) ANOVA with post hoc test. ${ }^{\star} P<0.05 ;{ }^{\star \star} P<0.001 ;$ n.s., $P \geq 0.05$. 
a

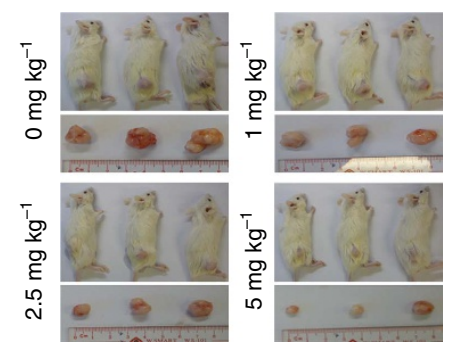

C

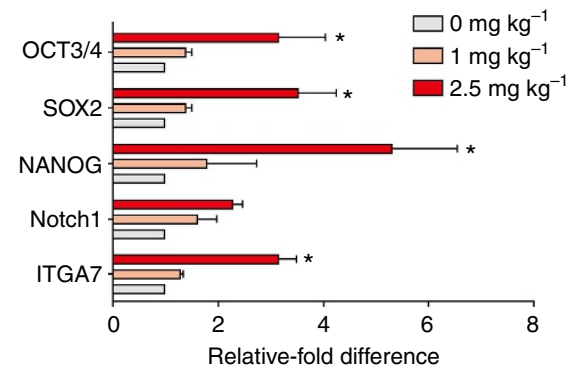

e

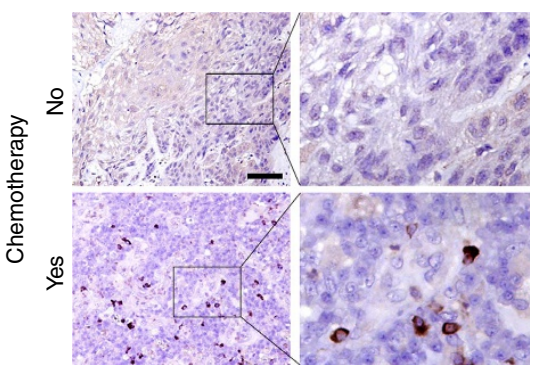

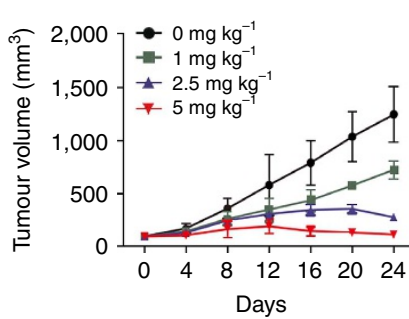

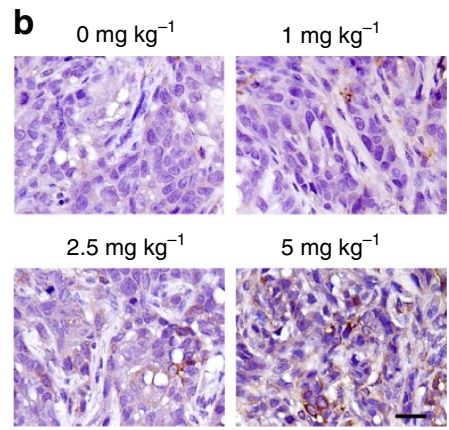

d
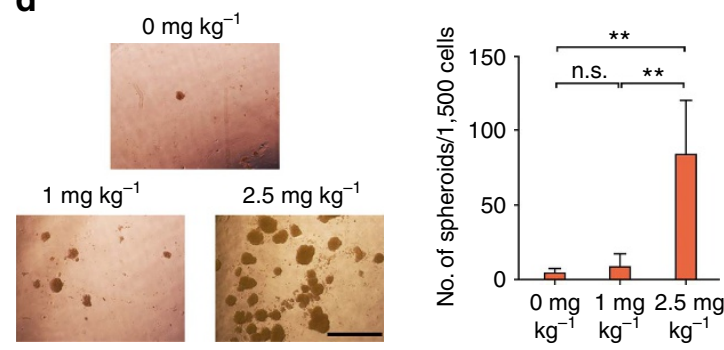

OSCC clinical samples

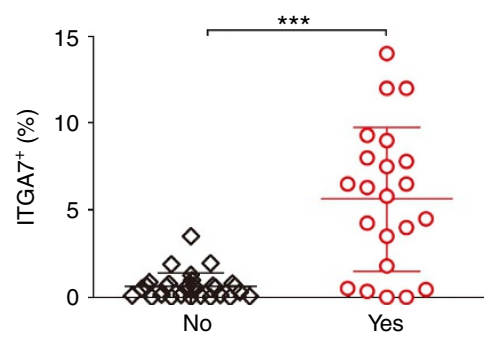

$\mathbf{f}$ K520 A7 $7^{-}$K520 A7 ${ }^{+} \quad$ K30-EV K30-A7 K520-NTC K520-shA7

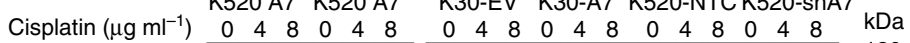

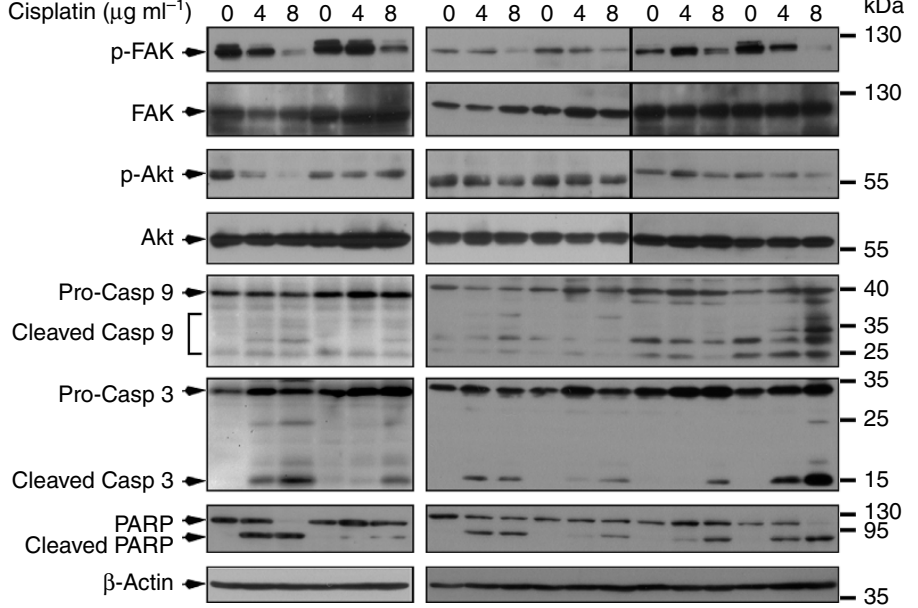

g CON

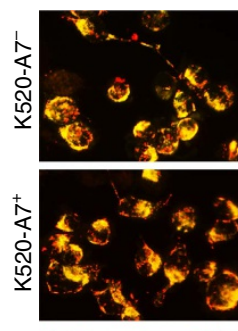

Cisplatin
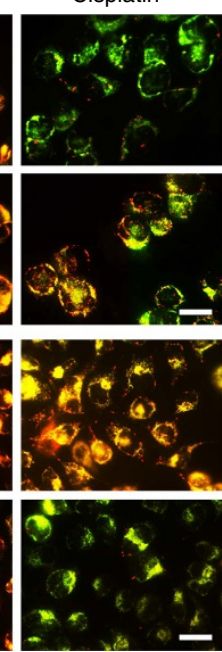

Figure 7 | ITGA7 promotes chemoresistance via the FAK/Akt/Caspase-9/Caspase-3 pathway. (a) NOD/SCID mice with xenograft tumours of 5 mm in diameter were randomly separated into four groups and treated with varying doses of cisplatin. The doses of 2.5 and $5 \mathrm{mg} \mathrm{kg} \mathrm{m}^{-1}$ cisplatin could effectively suppress tumour growth and shrink tumour size. The average tumour volume was expressed as the mean \pm s.d. of three mice. (b) Representative IHC images showing that $\mathrm{ITGA7}^{+}$cells were positively correlated with the dosage of cisplatin. Scale bar, $20 \mu \mathrm{m}$. (c) qRT-PCR showed that the expression of ITGA7 and stemness-associated genes was positively correlated with the dosage of cisplatin. (d) Representatives and summary of spheroid formation assay showing that isolated cells from chemoresistant group displayed enhanced self-renewal ability. Scale bar, $500 \mu \mathrm{m}$. (e) Representative images of ITGA7 IHC staining of clinical OSCC specimens had or had not undergone chemotherapy treatment. Compared with OSCC specimens without treatment $(0.64 \% \pm 0.74 \% ; n=28), I_{T G A}+$ cells were markedly increased after treatment $(5.64 \% \pm 4.15 \% ; n=22)$. Scale bar, $100 \mu \mathrm{m}$. (f) Western blot analysis was used to detect indicated protein levels in given cells $24 \mathrm{~h}$ after cisplatin treatment. (g) Representative images of JC-1 dye staining $24 \mathrm{~h}$ after cisplatin $\left(4 \mu \mathrm{g} \mathrm{ml}^{-1}\right.$ ) treatment. JC-1 dye aggregates at high $\Delta \Psi \mathrm{m}$ mitochondria (red) or forms monomers at low $\Delta \Psi_{\mathrm{m}}$ mitochondria (green). Scale bar, $50 \mu \mathrm{m}$. Statistics: (c,d) ANOVA with post hoc test. (e) Student $t$-test. ${ }^{\star} P<0.05$; ${ }^{\star \star} P<0.001$; ${ }^{\star \star \star} P<0.0001$; n.s., $P \geq 0.05$. 
CSCs? Another question is the heterogeneity of CSCs in a given cancer. For example, surface markers CD90 (ref. 10), CD44 (ref. 12) and $\mathrm{p}^{\mathrm{NTR}}$ (ref. 13), have been used for CSCs isolation in OSCC. Does this suggest that different CSC subsets may be derived from different cell of origins, or just in different hierarchical levels? The key issue behind these questions is to identify one specific protein that plays role in regulating stemness and CSC maintenance. Ideally, the optimized marker for CSC should have a function in maintaining CSC in a stem cell state, therefore, the marker should not be lost from the CSC without losing the stem cell properties. As a result, focus has been placed on enzymes or proteins, which can directly associate with CSC maintenance, such as ALDH1 (ref. 23) and ABCB5 (ref. 24). The identification of functional CSC markers has also focused on signalling pathways with an indispensable role in normal stem cell biology, such as Hedgehog ${ }^{25}$ and integrin signalling ${ }^{19-21}$.

In the present study, we aimed to identify a novel functional CSC marker in OSCC. Non-CG methylation has been confined as a characteristic of stem cells ${ }^{14,15}$, and several essential stemnessassociated genes, such as NANOG, SOX2 and OCT3/4 have been found highly modified with non-CG methylation in stem cells. It suggests that non-CG methylation may regulate transcriptional activity only in CSCs, but not in non-CSC. Here, we found that non-CG methylation preferentially presented in $\mathrm{ITGA}^{+}$rather than ITGA7 $^{-}$OSCC cells, implying that the transcriptional activity of ITGA7 might be also regulated by non-CG methylation. ITGA7 gene encodes for an ECM receptor integrin $\alpha 7$ that belongs to the integrin family. Recent studies indicate that some integrin family members, such as integrin $\alpha 6$ (ref. 19) and $\beta 1$ (ref. 26), play critical roles in maintaining stem cell niche, preserving a stable stem cell population, and regulating stem cell homoeostasis $^{27}$. In clinical OSCC specimens, ITGA7 ${ }^{+}$cells were expressed in $<1 \%$ of tumour cells in most tumour tissues $(203 / 262,77.5 \%)$, which was consistent with CSC expectation. Interestingly, high-frequency $\mathrm{ITGA}^{+}$cells $(>0.6 \%)$ was significantly associated with OSCC stemness such as cell differentiation, advanced clinical stage, tumour invasion and lymph node metastasis, suggesting that ITGA7 might play roles in regulating stemness.

Compared with a known oesophageal CSC marker CD90, the population of $\mathrm{ITGA}^{+}$cells was much smaller than the population of $\mathrm{CD}^{+} 0^{+}$cells in both immortalized oesophageal and OSCC cell lines. Interestingly, most of ITGA7 ${ }^{+}$cells were co-expressed with CD90, while only part of $\mathrm{CD}^{+} 0^{+}$cells co-expressed with ITGA7 in OSCC cell lines. Functional study found that $\mathrm{ITGA7}{ }^{+} / \mathrm{CD} 90^{+}$cells possessed stronger stemness compared with $\mathrm{ITGA7}^{-} / \mathrm{CD} 0^{+}$cell, implying that $\mathrm{ITGA}^{+}$ cells might be more properly represent CSCs in OSCC. Further functional assays were used to characterize ITGA7 $^{+}$cells sorted from KYSE180 and KYSE520 cells. Compared with ITGA7 $^{-}$ cells, ITGA7 ${ }^{+}$cells showed stronger abilities for self-renew, cell differentiation, cell motility and chemoresistance, as well as with higher expression of stemness-associated genes. In addition, ITGA7-transfected cells also possessed CSC properties. We also found ITGA7 ${ }^{+}$OSCC cells underwent EMT, which is responsible for initiation of metastasis ${ }^{28,29}$. All these CSC properties could be effectively inhibited when ITGA7 was silenced by shRNA. All these findings suggest that ITGA7 is a better CSC marker with stemness regulatory function. As expression level of ITGA7 was extremely low in clinical specimens, these functional assays could not be repeated in clinical samples. Further study found that the effects of ITGA7 on OSCC development might be through the activation of FAK/MAPK/ERK signalling pathway, which has been reported to be involved in a series of tumour processes $^{30}$. Similar to ITGA7 knockdown, FAK inhibitor could also inhibit the effect of ITGA7 on self-renewal ability and decrease the expression of stemness-associated genes such as OCT3/4, SOX2 and NANOG. Recently, Golubovskaya ${ }^{31}$ reported the interaction of FAK, NANOG, OCT3/4 and SOX2 played a critical role in regulating CSCs. This suggests that ITGA7 might be involved in stemness regulation and CSC maintenance in OSCC through the activation of FAK/MAPK/ERK signalling pathway.

One of the important characteristics of CSCs in clinical point of view is its resistance to conventional chemotherapy. In the present study, we demonstrated that $\mathrm{ITGA}^{+}$cells were markedly enriched in residual OSCC xenograft tumours in NOD/SCID mice after treatment with cisplatin chemotherapy. Consistent with this data, the enrichment of ITGA7 ${ }^{+}$cells was also observed in clinical OSCC samples after chemotherapy. This finding may help explain why chemotherapy fails to eradicate the disease although it can effectively shrink the tumour size by killing most of tumour cells. Therefore, development of novel therapeutic strategy by targeting CSCs is necessary to cure cancer. Further study found that ITGA7 exerted the anti-apoptotic effect through the versatile player FAK to activate the Akt signalling, which subsequently inhibited the release of cytochrome $c$ into the cytoplasm and the cleavages of caspase-9, caspase-3 and PARP. In summary, we report here that ITGA7 is a potential CSC marker in OSCC with function in stemness regulation and CSCs maintenance. Further characterization of ITGA7 may provide an alternative therapeutic approach by targeting CSCs to achieve better clinical outcome.

\section{Methods}

OSCC clinical specimens. Three hundred pairs of primary OSCC specimens and their adjacent non-tumour oesophageal tissues, used for a TMA, were collected from patients who underwent oesophageal resection at Linzhou Cancer hospital (Henan, China). Twenty-eight clinical specimens were collected from OSCC patients at Sun Yat-Sen University Cancer Center (Guangzhou, China). None of these patients received preoperative treatment. Twenty-two clinical specimens were collected from patients with OSCCs who had received neoadjuvant chemoradiotherapy before operation at the Queen Mary Hospital (Hong Kong).

Histological examination was carried out by pathologists, and diagnosis was made based on the microscopic features of the carcinoma cells. Tumours were graded using the American Joint Committee on Cancer (AJCC)/International Union Against Cancer (UICC) tumour staging system. Informed consent was obtained from all patients before the collection of oesophageal specimens, and the study was approved by the committees for ethical review of research involving human subjects at Zhengzhou University (Zhengzhou, China), Sun Yat-Sen University (Guangzhou, China) and the University of Hong Kong (Hong Kong).

Cell lines. Immortalized oesophageal epithelial cell lines NE1 and NE3 were established in Professor George Tsao's laboratory (Department of Anatomy, The University of Hong Kong). Chinese OSCC cell lines HKESC1, EC18, EC109 and EC9706 were kindly provided by Professor Srivastava (Department of Pathology, The University of Hong Kong). Six Japanese OSCC cell lines (KYSE30, KYSE140, KYSE180, KYSE410, KYSE510 and KYSE520) were obtained from DAMZ, the German Resource Center for Biological Material. Mycoplasma contamination was not found in these cell lines.

Animals. The study protocol was approved by and performed in accordance with the Committee of the Use of Live Animals in Teaching and Research at the University of Hong Kong. For in vivo tumorigenic experiment, various numbers of ITGA7 sorted cells, ITGA7-transfected cells and ITGA7-suppressed cells were injected subcutaneously into 4-to-5-week-old NOD/SCID mice. Mice were killed between 2 and 4 months post injection. Those animals injected with tumour cells but no sign of tumour burden were generally terminated 4 months after tumour cell inoculation, and mice were opened up at the injection sites to confirm that there was no tumour development. For in vivo metastasis assay, each experimental group consisted of seven 5 -week-old NOD/SCID mice. Briefly, $2 \times 10^{5}$ cells in $20 \mu \mathrm{l}$ phosphate-buffered saline (PBS) were injected into the right hind foot-pad of each mouse. All of the mice were euthanized 4 weeks after injection. The right side popliteal lymph nodes were excised and embedded in paraffin. For chemoresistant xenograft model, subcutaneous xenografts in 5-week-old NOD/SCID mice were established with KYSE520 cells $\left(1 \times 10^{6}\right)$. Treatment was started once the size of the xenograft reached $\sim 5 \mathrm{~mm}$ in diameter. The mice were randomly assigned into four groups, each consisting of three mice. They were treated with cisplatin intraperitoneally every 4 days for 24 days at different doses of $0,1,2.5$ or 
$5 \mathrm{mg} \mathrm{kg}^{-1}$. Tumour sizes were measured by caliper, and tumour volumes were calculated as volume $\left(\mathrm{mm}^{3}\right)=L \times W^{2} \times 0.5$. Cell dissociation from xenograft is detailed in Supplementary Methods.

IHC. IHC staining was performed using the standard streptavidin-biotinperoxidase complex method. Briefly, paraffin sections were deparaffinized and rehydrated. Slides were heated for antigen retrieval for $15 \mathrm{~min}$ in $10 \mathrm{mM}$ citrate $(\mathrm{pH}$ ). Sections were incubated with monoclonal mouse anti-human ITGA7 (1:100 dilution; Abgent) at $4{ }^{\circ} \mathrm{C}$ overnight. EnVision Plus System-HRP (DAB; DAKO) was used according to manufacturer's instruction, and followed by Mayer's hematoxylin counterstaining. Stained slides were imaged on an AperioScanscope CS imager (Vista, CA, USA).

Immunofluorescence. Immunofluorescence was performed on cell lines and cryosectioned spheroids. First, cells on the coverslips were fixed with $4 \%$ paraformaldehyde, and incubated with primary antibody (rabbit anti-ITGA7 or mouse anti-CD90) overnight at $4{ }^{\circ} \mathrm{C}$. After washing, cells underwent incubation with FITC- or PE-conjugated secondary antibodies for $1 \mathrm{~h}$, and subsequently counterstained with DAPI for $5 \mathrm{~min}$ at room temperature (Roche Diagnostics). All images were visualized under Leica DMRA fluorescence microscope (Wetzlar, Germany).

Flow cytometry and cell sorting. Flow cytometric analysis and cell sorting was conducted using anti-human ITGA7 (Abgent), and PE-conjugated mouse anti-human CD90 (BD Biosciences). Cells were gently detached in citric saline buffer, and incubated in PBS containing $2 \%$ fetal bovine serum (FBS) with either PE-conjugated primary antibody or primary antibody followed by a FITC-conjugated secondary antibody. Isotype-matched mouse or rabbit immunoglobulin served as controls to gate positive cells. Samples were analyzed and sorted on BD LSR Fortessa Analyzer and FACSAria I Cell Sorter (BD Biosciences), and data analyzed using FlowJo software (Tree Star). For ITGA7 cell sorting, only the top $10 \%$ most brightly stained or the bottom $15 \%$ most dimly stained cells were gated as positive and negative populations, respectively. Cell viability was assessed using trypan blue exclusion. Using antibodies listed in Supplementary Table 4.

Western blot analysis. Quantified protein lysates were resolved on SDS-PAGE, transferred onto a polyvinylidenedifluoride (PVDF) membrane (Millipore), and then blocked with 5\% non-fat milk in Tris-buffered saline-Tween 20 (TBS-T) for $1 \mathrm{~h}$ at room temperature. The blocked membrane was then incubated with primary antibody at $4{ }^{\circ} \mathrm{C}$ overnight. After washing with TBS-T, the membrane was incubated for $1 \mathrm{~h}$ with horseradish peroxidase (HRP)-conjugated secondary antibody. A complex of primary and secondary antibodies-labelled proteins were detected by enhanced chemiluminescence (ECL) system (GE Healthcare) and $\mathrm{X}$-ray film (GE Healthcare). $\beta$-actin was used as a housekeeping control. Using antibodies listed in Supplementary Table 4. Full blots of Figs $3 \mathrm{c}$ and $7 \mathrm{f}$ are provided in Supplementary Fig. 6.

DNA extraction and non-CG methylation analysis. Genomic DNA was extracted from isolated ITGA7 ${ }^{+}$and ITGA7 ${ }^{-}$cells by phenol-chloroform method followed by bisulfite modification using the EpiTECT Bisulfite Kit (Qiagen). BGS primers were listed in Supplementary Table 5. The template was amplified by PCR for 35 cycles and then cloned into the pGEM-T Easy vector (Promega) and sequenced as individual clones by Beijing Genomics Institute. The sequence data was analyzed by BiQ analyzer (Max Planck Institute).

RNA extraction and quantitative real-time PCR. Total RNA was extracted using TRIZOL Reagent (Invitrogen), and CDNA was synthesized using a reverse transcription (RT)-PCR Kit (Roche) according to the manufacturer's instructions. Quantitative real-time PCR (qRT-PCR) was performed using the SYBR Green PCR Kit (Applied Biosystems) and an ABI PRISM 7900 Sequence Detector (Applied Biosystems). Specificity of primers was verified by dissociation curve analysis. Data was analyzed using ABI SDS v2.4 software (Applied Biosystems). All qRT-PCR reactions were performed in duplicates. Housekeeping gene GAPDH was used as an internal control. Using primers listed in Supplementary Table 5.

Spheroid formation assay. Single cells were cultured in suspension in 24-well polyHEMA (Sigma-Aldrich)-coated plates. Cells were grown in $300 \mu \mathrm{l}$ of serum-free DMEM/F12 medium (Invitrogen) supplemented with $4 \mu \mathrm{g} \mathrm{ml}^{-1}$ insulin (Sigma-Aldrich), B27 (1:50; Invitrogen), $20 \mathrm{ng} \mathrm{ml}^{-1}$ human recombinant EGF (Sigma-Aldrich), $10 \mathrm{ng} \mathrm{ml}^{-1}$ human recombinant basic FGF (Invitrogen). Cells were replenished with $30 \mu \mathrm{l}$ of supplemented medium every second day. For serial passage of primary spheroids, the primary spheroids were collected and dissociated to single cells using TrypLE Expression (Invitrogen). Following dissociation, trypsin inhibitor (Invitrogen) was used to neutralize the reaction, and cells were resuspended in DMEM/F12 medium with the above supplements.
Apoptosis assay. Cells were treated with cisplatin or 5-FU (KYSE180 with $6 \mu \mathrm{g} \mathrm{ml}^{-1}$ cisplatin or $12 \mu \mathrm{g} \mathrm{ml}^{-1}$ 5-FU; KYSE520 with $10 \mu \mathrm{g} \mathrm{ml}^{-1}$ cisplatin or $30 \mu \mathrm{g} \mathrm{ml}^{-1}$ 5-FU; EC109 with $4 \mu \mathrm{g} \mathrm{ml}^{-1}$ cisplatin or $20 \mu \mathrm{g} \mathrm{ml}^{-1} 5$-FU; and KYSE30 with $1 \mu \mathrm{g} \mathrm{ml}^{-1}$ cisplatin or $5 \mu \mathrm{g} \mathrm{ml}^{-1}$ 5-FU) for $48 \mathrm{~h}$. Cells then were collected and stained with propidium iodide (PI), and FITC-conjugated Annexin-V provided by the Annexin-V FLUOS Staining Kit (Roche Diagnostics) according to manufacturer's instructions. Analysis was determined by flow cytometry on a LSR Fortessa Analyzer (BD Biosciences) and FlowJo software (Tree Star).

Differentiation assay. atRA (listed in Supplementary Table 6) was used to treat sorted ITGA7 ${ }^{-}$and ITGA7 ${ }^{+}$cells at a concentration of 20 or $30 \mu \mathrm{M}$ in RPMI 1640 medium supplemented with $10 \%$ FBS and $1 \%$ penicillin and streptomycin for 5 days, with medium change daily. Expression change of ITGA7, differentiation markers CK13 and CK14, and stemness-associated genes, was assessed by western blot analysis.

In vitro tumorigenic assays. Anchorage-dependent growth was assessed by foci formation assay. Briefly, $2 \times 10^{3}$ cells were seeded in a 6 -well plate. Surviving colonies ( $>50$ cells per colony) were stained with $1 \%$ crystal violet (Sigma-Aldrich) and counted after 1 week. Anchorage-independent growth was assessed by colony formation in soft agar. In brief, $5 \times 10^{3}$ cells mixed thoroughly in $0.35 \%$ low melting soft agar (BD Biosciences) were grown in a 6-well plate precoated with $0.5 \%$ agar. After 2 weeks, colonies ( $\geq 10$ cells) were counted under microscope in 10 fields per well and photographed.

ITGA7 overexpression and knockdown. ITGA7 overexpression plasmid was kindly provided by Dr. Jianhua Luo (Department of Pathology, University of Pittsburgh School of Medicine). Briefly, the full-length wild-type ITGA7 cDNA (NM_002206.2) was cloned into the pLenti6 expression vector (Invitrogen) and transfected into EC109 and KYSE30 cells with Lipofectamine 2000 Reagent (Invitrogen). Stable ITGA7-expressing clones were selected for 2 weeks with blasticidin (Sigma-Aldrich). EV-transfected cells were used as controls. For ITGA7 knockdown, the scrambled shRNA plasmid (pLKO.1-NTC) and the ITGA7-specfic shRNA expression vectors (pLKO.1-shITGA7) were purchased from Sigma-Aldrich Two constructs against ITGA7 were used: shITGA7-1 (TRCN0000057708) and shITGA7-3 (TRCN0000057711). The shRNA sequences listed in Supplementary Table 7. The pLKO.1-shITGA7 or the scrambled shRNA plasmid was transfected into KYSE180 and KYSE520, and stable clones were selected using puromycin (Sigma-Aldrich).

Migration and invasion assays. Migration and invasion assays were performed in 24-well millicell hanging insert (Millipore) or 24-well BioCoat Matrigel Invasion Chambers (BD Biosciences). In brief, $1 \times 10^{5}$ cells were seeded to the top chamber and $10 \%$ FBS in medium was added to the bottom chamber as a chemoattractant. After 24 or 48-h incubation, the number of cells that invaded through the membrane (migration) or Matrigel (invasion) was counted in 10 fields and imaged using SPOT imaging software (Nikon).

Mitochondrial membrane potential assay. The mitochondrial membrane potential $\left(\Delta \Psi_{\mathrm{m}}\right)$ was detected with a MitoPTJC-1 detection kit (Immunochemistry Technologies) according to the manufacturer's protocol. In brief, cells were seeded onto coverslips and cultured to $80 \%$ confluence; then $4 \mu \mathrm{g} \mathrm{ml}^{-1}$ cisplatin was added to treat cells for $24 \mathrm{~h}$. The cells were washed twice with PBS and incubated with the $\Delta \Psi_{\mathrm{m}}$-sensitive dye JC-1 at $37^{\circ} \mathrm{C}$ for $15 \mathrm{~min}$. Images were visualized immediately under Leica DMRA fluorescence microscope (Wetzlar, Germany).

Statistics. Statistical analysis was carried out with SPSS v. 16 (Chicago, IL, USA). Pearson $\chi^{2}$ test was used to analyze the association of ITGA7 expression with clinicopathologic parameters. Kaplan-Meier plot and log-rank test were used for survival analysis. Independent Student $t$-test and ANOVA with post hoc test were used for most studies as indicated in the figure legends. For limiting dilution assay, the tumour-initiating frequency and statistics were calculated using ELDA software ${ }^{32}$. The data are presented as the mean \pm s.d. of three independent experiments. The $P$-values were denoted as ${ }^{*} P<0.05,{ }^{*} P<0.001$ and ${ }^{* * *} P<0.0001$ in all figures.

Data availability. The data that support the findings of this study are available from the corresponding author upon request.

\section{References}

1. Ferlay, J. et al. Estimates of worldwide burden of cancer in 2008: GLOBOCAN 2008. Int. J. Cancer 127, 2893-2917 (2010).

2. Li, J. Y. Epidemiology of esophageal cancer in China. Natl. Cancer Inst. Monogr. 62, 113-120 (1982). 
3. Ma, S., Lee, T. K., Zheng, B. J., Chan, K. W. \& Guan, X. Y. CD133 + HCC cancer stem cells confer chemoresistance by preferential expression of the Akt/PKB survival pathway. Oncogene 27, 1749-1758 (2008).

4. Pinto, C. A., Widodo, E., Waltham, M. \& Thompson, E. W. Breast cancer stem cells and epithelial mesenchymal plasticity-implications for chemoresistance. Cancer Lett. 341, 56-62 (2013).

5. Elshamy, W. M. \& Duhe, R. J. Overview: cellular plasticity, cancer stem cells and metastasis. Cancer Lett. 341, 2-8 (2013).

6. Adorno-Cruz, V. et al. Cancer stem cells: targeting the roots of cancer, seeds of metastasis, and sources of therapy resistance. Cancer Res. 75, 924-929 (2015).

7. Hanahan, D. \& Weinberg, R. A. Hallmarks of cancer: the next generation. Cell 144, 646-674 (2011).

8. Al-Hajj, M., Wicha, M. S., Benito-Hernandez, A., Morrison, S. J. \& Clarke, M. F. Prospective identification of tumorigenic breast cancer cells. Proc. Natl Acad. Sci. USA 100, 3983-3988 (2003).

9. Ma, S. et al. Identification and characterization of tumorigenic liver cancer stem/progenitor cells. Gastroenterology 132, 2542-2556 (2007).

10. Tang, K. H. et al. A CD90 $(+)$ tumour-initiating cell population with an aggressive signature and metastatic capacity in esophageal cancer. Cancer Res. 73, 2322-2332 (2013).

11. Tong, C. M., Ma, S. \& Guan, X. Y. Biology of hepatic cancer stem cells. J. Gastroenterol. Hepatol. 26, 1229-1237 (2011).

12. Zhao, J. S. et al. Tumor initiating cells in esophageal squamous cell carcinomas express high levels of CD44. PLoS ONE 6, e21419 (2011).

13. Okumura, T. et al. The biological role of the low-affinity $\mathrm{p} 75$ neurotrophin receptor in esophageal squamous cell carcinoma. Clin. Cancer Res. 12, 5096-5103 (2006).

14. Lister, R. et al. Human DNA methylomes at base resolution show widespread epigenomic differences. Nature 462, 315-322 (2009).

15. Ramsahoye, B. et al. Non-CpG methylation is prevalent in embryonic stem cells and may be mediated by DNA methyltransferase 3a. Proc. Natl Acad. Sci. USA 97, 6 (2000).

16. Reya, T., Morrison, S. J., Clarke, M. F. \& Weissman, I. L. Stem cells, cancer, and cancer stem cells. Nature 414, 105-111 (2001).

17. Pardal, R., Clarke, M. F. \& Morrison, S. J. Applying the principles of stem-cell biology to cancer. Nat. Rev. Cancer 3, 895-902 (2003).

18. Desgrosellier, J. S. \& Cheresh, D. A. Integrins in cancer: biological implications and therapeutic opportunities. Nat. Rev. Cancer 10, 9-22 (2010).

19. Lathia, J. D. et al. Integrin alpha 6 regulates glioblastoma stem cells. Cell Stem Cell 6, 421-432 (2010).

20. Lo, P. K. et al. CD49f and CD61 identify Her2/neu-induced mammary tumorinitiating cells that are potentially derived from luminal progenitors and maintained by the integrin-TGFbeta signaling. Oncogene 31, 2614-2626 (2012).

21. Yoshioka, T. et al. beta4 Integrin signaling induces expansion of prostate tumor progenitors. J. Clin. Invest. 123, 682-699 (2013).

22. Qian, C. N. et al. Preparing the "soil": the primary tumor induces vasculature reorganization in the sentinel lymph node before the arrival of metastatic cancer cells. Cancer Res. 66, 10365-10376 (2006).

23. Ginestier, C. et al. ALDH1 is a marker of normal and malignant human mammary stem cells and a predictor of poor clinical outcome. Cell Stem Cell 1, 555-567 (2007).

24. Schatton, T. et al. Identification of cells initiating human melanomas. Nature 451, 345-349 (2008).

25. Liu, S. et al. Hedgehog signaling and Bmi-1 regulate self-renewal of normal and malignant human mammary stem cells. Cancer Res. 66, 6063-6071 (2006).

26. Veevers-Lowe, J., Ball, S. G., Shuttleworth, A. \& Kielty, C. M. Mesenchymal stem cell migration is regulated by fibronectin through alpha5betal-integrinmediated activation of PDGFR-beta and potentiation of growth factor signals. J. Cell Sci. 124, 1288-1300 (2011).

27. Brizzi, M. F., Tarone, G. \& Defilippi, P. Extracellular matrix, integrins, and growth factors as tailors of the stem cell niche. Curr. Opin. Cell Biol. 24, 645-651 (2012).
28. Brabletz, T. EMT and MET in metastasis: where are the cancer stem cells? Cancer Cell 22, 699-701 (2012).

29. Mani, S. A. et al. The epithelial-mesenchymal transition generates cells with properties of stem cells. Cell 133, 704-715 (2008)

30. Tong, M. et al. Rab25 is a tumor suppressor gene with antiangiogenic and anti-invasive activities in esophageal squamous cell carcinoma. Cancer Res. 72, 6024-6035 (2012).

31. Golubovskaya, V. M. FAK and Nanog cross talk with p53 in cancer stem cells. Anticancer Agents Med. Chem. 13, 576-580 (2013).

32. Hu, Y. \& Smyth, G. K. ELDA: extreme limiting dilution analysis for comparing depleted and enriched populations in stem cell and other assays. J. Immunol. Methods 347, 70-78 (2009).

\section{Acknowledgements}

We thank the Faculty Core Facility of the LKS Faculty of Medicine at The University of Hong Kong for help with the flow cytometry cell sorting, as well as Dr. Jianhua Luo (Department of Pathology, University of Pittsburgh School of Medicine) for providing the ITGA7-expressing plasmid. This work was supported by grants from the Hong Kong Research Grant Council (RGC) grants including Collaborative Research Funds (C7038-14G and C7027-14G), General Research Funds (HKU/ 7668/11M, 767313 and CUHK/766613), NSFC/RGC Joint Research

Scheme (N_HKU712/12), Hong Kong Health and Medical Research Found (02133366), grants from the National Basic Research Program of China (2012CB967001), China National Key Sci-Tech Special Project of Infectious Diseases (2013ZX10002-011-005), National Natural Science Foundation of China (81272416 and 81372583) and the Science and Technology Foundation of Shenzhen (CXZZ20150430092951135 and KQTD20140630100658078). Professor X.Y. Guan is Sophie YM Chan Professor in Cancer Research.

\section{Author contributions}

Conceptualization: X.-Y.M., L.F. and X.-Y.G.; methodology: X.-Y.M., L-Y.Z. and X.-Y.G.; investigation: X.-Y.M., L-Y.Z. and T-T.C.; writing-original drafts: X.-Y.M.; Writing—review \& editing: X.-Y.M., S.M. and X.-Y.G.; funding acquisition: L.F. and X.-Y.G.; resources: Y-R.Q., K.W.C., S.M. and D.X. and supervision: X.-Y.G.

\section{Additional information}

Supplementary Information accompanies this paper at http://www.nature.com/ naturecommunications

Competing financial interests: The authors declare no competing financial interests.

Reprints and permission information is available online at http://npg.nature.com/ reprintsandpermissions/

How to cite this article: Ming, X.-Y. et al. Integrin $\alpha 7$ is a functional cancer stem cell surface marker in oesophageal squamous cell carcinoma. Nat. Commun. 7, 13568 doi: $10.1038 /$ ncomms13568 (2016).

Publisher's note: Springer Nature remains neutral with regard to jurisdictional claims in published maps and institutional affiliations.

This work is licensed under a Creative Commons Attribution 4.0 International License. The images or other third party material in this article are included in the article's Creative Commons license, unless indicated otherwise in the credit line; if the material is not included under the Creative Commons license, users will need to obtain permission from the license holder to reproduce the material To view a copy of this license, visit http://creativecommons.org/licenses/by/4.0/

(C) The Author(s) 2016 